\title{
A Novel Human Pluripotent Stem Cell-Derived Neural Crest Model of Treacher Collins Syndrome Shows Defects in Cell Death and Migration
}

\author{
Felipe Serrano,, ${ }^{1, *}$ William George Bernard, ${ }^{1, *}$ Alessandra Granata, ${ }^{2}$ Dharini lyer, ${ }^{1}$ Ben Steventon, ${ }^{3}$ \\ Matthew Kim, ${ }^{1}$ Ludovic Vallier, ${ }^{1}$ Laure Gambardella, ${ }^{1}$ and Sanjay Sinha ${ }^{1}$
}

The neural crest (NC) is a transient multipotent cell population present during embryonic development. The NC can give rise to multiple cell types and is involved in a number of different diseases. Therefore, the development of new strategies to model NC in vitro enables investigations into the mechanisms involved in NC development and disease. In this study, we report a simple and efficient protocol to differentiate human pluripotent stem cells (HPSC) into NC using a chemically defined media, with basic fibroblast growth factor 2 (FGF2) and the transforming growth factor- $\beta$ inhibitor SB-431542. The cell population generated expresses a range of NC markers, including P75, TWIST1, SOX10, and TFAP2A. NC purification was achieved in vitro through serial passaging of the population, recreating the developmental stages of $\mathrm{NC}$ differentiation. The generated NC cells are highly proliferative, capable of differentiating to their derivatives in vitro and engraft in vivo to NC specific locations. In addition, these cells could be frozen for storage and thawed with no loss of NC properties, nor the ability to generate cellular derivatives. We assessed the potential of the derived NC population to model the neurocristopathy, Treacher Collins Syndrome (TCS), using small interfering RNA (siRNA) knockdown of TCOF1 and by creating different TCOF $1^{+-}$HPSC lines through CRISPR/Cas 9 technology. The NC cells derived from TCOF ${ }^{+/-}$HPSC recapitulate the phenotype of the reported TCS murine model. We also report for the first time an impairment of migration in $\mathrm{TCOF}^{+1-} \mathrm{NC}$ and mesenchymal stem cells. In conclusion, the developed protocol permits the generation of the large number of $\mathrm{NC}$ cells required for developmental studies, disease modeling, and for drug discovery platforms in vitro.

Keywords: neural crest, differentiation, disease modeling, Treacher Collins syndrome, human pluripotent stem cells

\section{Introduction}

I N MAMMALIAN DEVELOPMENT, neural crest (NC) cells are a transient multipotent population arising from the neural plate border, usually contemporaneously with neural tube closure [1]. These migratory cells invade adjacent tissues and differentiate into multiple cell types, including mesenchymal stem cells (MSC), vascular smooth muscle cells (SMC), adipocytes, osteocytes, chondrocytes, melanocytes, glia, and Schwann cells [2]. NC cells are also implicated in a broad range of pathologies making the in vitro generation of these cells of high clinical relevance.
The development and function of the NC have been well characterized in animal models, including the chick, zebrafish, and mouse [3-6]. NC specification and induction depend on signaling molecules and transcription factors, whose actions are coordinated during gastrulation and neurulation. The neural plate border is specified by the cross talk between BMP, WNT, and fibroblast growth factor (FGF) signaling [7]. Initially, intermediate levels of BMP signaling induce neural folds with anterior character over the whole neural plate border. Next, the posterior regions of the neural plate border are transformed into $\mathrm{NC}$ under the effects of FGF, WNT, and retinoic acid [8].

\footnotetext{
${ }^{1}$ Anne McLaren Laboratory, Wellcome Trust-Medical Research Council Cambridge Stem Cell Institute, Department of Medicine, University of Cambridge, Cambridge, United Kingdom.

${ }^{2}$ Division of Clinical Neurosciences, Clifford Allbutt Building, Cambridge Biomedical Campus, Cambridge, United Kingdom.

${ }^{3}$ Department of Genetics, University of Cambridge, Cambridge, United Kingdom.

*These authors contributed equally to this work.

(C) Felipe Serrano et al. 2018; Published by Mary Ann Liebert, Inc. This Open Access article is distributed under the terms of the Creative Commons License (http://creativecommons.org/licenses/by/4.0), which permits unrestricted use, distribution, and reproduction in any medium, provided the original work is properly cited.
} 
After specification, NC cells undergo epithelial-mesenchymal transition (EMT) before delaminating from the border of the neuroepithelium [9]. Subsequently the arising NC cells migrate in response to specific cues and express specific proteins, including Twist [10], Sox10 [3,11], and p75 [12]. These factors control cellular events such as delamination, cell proliferation, migration, and differentiation [13-15]. Defects in the complex processes that choreograph $\mathrm{NC}$ development are involved in congenital human diseases known as neurocristopathies.

Human embryonic stem cells (HESC) and human induced pluripotent stem cells (HIPSC) are collectively referred to as human pluripotent stem cells (HPSC). Some of the most relevant NC differentiation protocols from HPSC are based on initially producing in vitro neuroectoderm [16-18] and then purifying the nascent NC population using different approaches such as dual SMAD inhibition [18-21] and/or WNT pathway activation [22-24]. A common limitation of these approaches is the use of undefined basement membrane extracts as a coating for cell adhesion, while only a few protocols have so far been validated in vivo [21,24-26]. Further optimization and validation of NC in vitro differentiation protocols would be valuable for studies into human development and disease.

Treacher Collins Syndrome (TCS) [27,28] (OMIM: $154500)$ is a neurocristopathy resulting in a severe congenital craniofacial disorder, which occurs one in every 50,000 births [29]. TCS is primarily associated with autosomal dominant haploinsufficiency-inducing mutations in the TCOF1 gene located on chromosome 5 [30], which lead to deficient ribosome biogenesis [31]. Tcofl is expressed broadly throughout the mouse embryo, with high activity in the neuroepithelium where it plays an essential role in cell survival [32]. Extensive apoptosis of the neuroepithelial progenitor has been reported in TCS, resulting in impaired NC differentiation and subsequent defects in craniofacial development [32].

In this study, we report a complete differentiation protocol using simple conditions that permits the generation of the NC from HPSC using a combination of FGF signaling and TGF- $\beta$ inhibition. Derived NC cells are proliferative, can be maintained over multiple passages, can differentiate to a variety of cell types in vitro, and have been validated in a developmental chick embryo model. Furthermore, we have utilized CRISPR/ Cas9 technology to generate $T C O F 1^{+/-}$lines as a model of TCS and have revealed abnormalities in cell migration, which may play a role in the underlying pathology of the disease. The development of this protocol permits the generation of $\mathrm{NC}$ and its derivatives in a chemically defined media (CDM) for developmental studies, disease modeling, and drug discovery.

\section{Materials and Methods}

\section{HPSC culture}

HESCs (H9s line; WiCell, Madison, WI) and HIPSC (BBHX8 line [33], Cambridge Biomedical Research Centre HIPSC core facility) called together as HPSC were maintained with CDM plus bovine serum albumin fraction $\mathrm{A}$ (CDM-BSA), as previously described [34]. CDM-BSA was composed of Iscove's modified Dulbecco's medium (Thermo Fisher Scientific) plus Ham's F12 NUT-MIX (Thermo
Fisher Scientific) medium in a 1:1 ratio, supplemented with GlutaMAX-I (Thermo Fisher Scientific), BSA (5 mg/mL; Europa Bioproducts), chemically defined lipid concentrate (Thermo Fisher Scientific), transferrin $(15 \mu \mathrm{g} / \mathrm{mL}$; Roche Diagnostics), insulin ( $7 \mu \mathrm{g} / \mathrm{mL}$; Roche Diagnostics), and monothioglycerol ( $450 \mu \mathrm{M}$; Sigma). For the maintenance of HPSC, CDM-BSA was supplemented with Activin A (10 ng/mL; R\&D Systems) and FGF2 (12 ng/mL; R\&D Systems), and cells were maintained on tissue culture treated plastic coated with $0.1 \%$ gelatin (Sigma-Aldrich).

HPSC were also cultured and maintained in $\mathrm{TeSR}^{\mathrm{TM}}-\mathrm{E}^{\mathrm{TM}}$ media (STEMCELL Technologies) using Vitronectin XF (STEMCELL Technologies) as chemically defined xenofree cell culture matrix.

\section{HPSC differentiation to NC}

For NC differentiation, HPSC were detached from gelatincoated plates using $1 \mathrm{mg} / \mathrm{mL}$ collagenase IV (Gibco). Clumps were triturated, counted, and plated at a density of 300 colonies/well in $0.1 \%$ gelatin-coated six well plates in CDM-BSA supplemented with Activin A (10 ng/mL; R\&D Systems) and FGF2 (12 ng/mL; R\&D Systems), referred to herein as CDM$\mathrm{BSA}+\mathrm{FA}$

After $24 \mathrm{~h}$ in CDM-BSA, the media was changed to CDM-polyvinyl alcohol (PVA) supplemented with FGF2 (12 ng/mL; R\&D Systems) and SB-431542 (10 $\mu \mathrm{M}$; Tocris), referred to herein as FSB, for 4 days without splitting. CDM-PVA has the same composition as CDM-BSA, with PVA ( $1 \mathrm{mg} / \mathrm{mL}$; Sigma) instead of BSA. On day 4 in FSB, the differentiating HPSC was dissociated using TrypLE Express $^{\text {TM }}$ (Gibco) and seeded as single cells at a 1:3 ratio on $0.1 \%$ gelatin-coated plates in FSB. The single cells were maintained in FSB (with daily media changes) on $0.1 \%$ gelatin-coated plates over several passages; the splitting of those single cells was performed every 3-4 days (80\%-90\% confluence) at 1:3 ratio using TrypLE Express (Gibco).

For differentiating HPSC grown on Vitronectin XF and maintained with TeSR-E8, the HPSC colonies were detached using 0.5 mM EDTA (Gibco). Clumps were counted and seeded at a density of 300 colonies/well in vitronectin XF-coated six well plates in TeSR-E8 media. After $24 \mathrm{~h}$ in TeSR-E8, the media was changed to FSB, and cells were maintained in FSB on vitronectin XF without splitting for 4 days. At day 4 in FSB, the differentiating HPSC were dissociated with TrypLE Express, seeded as single cells on $0.1 \%$ gelatin-coated plates and split every 3-4 days as described above.

\section{Quantitative real-time polymerase chain reaction}

Total RNA was extracted using the RNeasy Mini Kit (Qiagen). Complementary DNA (cDNA) was synthesized from $250 \mathrm{ng}$ total RNA using the Maxima First Strand cDNA Synthesis Kit (Thermo Fisher Scientific). Quantitative realtime polymerase chain reaction (qRT-PCR) mixtures were prepared with the FAST-SYBR Green Master Mix (Thermo Fisher Scientific) and analyzed on a 7500 Fast Real-time PCR system (Thermo Fisher Scientific). $\mathrm{C}_{\mathrm{T}}$ values were normalized to porphobilinogen deaminase (PBGD). Primer sequences are listed in Supplementary Table S1 (Supplementary Data are available online at www.liebertpub.com/scd). 


\section{Flow cytometry}

Cells were fixed with Cytofix/Cytoperm Fixation solution (BD Biosciences) for $20 \mathrm{~min}$ at $4^{\circ} \mathrm{C}$, then washed with Perm Wash Buffer/phosphate-buffered saline (PBS, $1 \times$; BD Biosciences) and permeabilized with Perm Wash Buffer/PBS $+0.1 \%$ Triton X-100 for $30 \mathrm{~min}$. Cells were blocked with $3 \%$ BSA in $1 \times$ Perm Wash Buffer at room temp for $30 \mathrm{~min}$. After blocking, cells were incubated in primary antibody (Supplementary Table S2) diluted in $1 \times$ Perm Wash Buffer $+0.1 \%$ Triton $\mathrm{X}-1004^{\circ} \mathrm{C}$ for $45 \mathrm{~min}$. Alexa Fluor ${ }^{\circledR}$-tagged secondary antibody was added after primary incubation for $1 \mathrm{~h}$ at room temperature. Samples were run on a Beckman Coulter CyAn-ADP flow cytometer, and subsequent datasets were analyzed using FlowJo software.

\section{Immunocytochemistry}

Adherent cells were fixed using 4\% PFA, permeabilized with $0.5 \%$ Triton X-100 in PBS (Sigma), and blocked with PBS $+3 \%$ BSA for $60 \mathrm{~min}$ at room temperature. Primary antibody (Supplementary Table S2) incubations were performed at $4^{\circ} \mathrm{C}$ overnight and Alexa Fluor tagged secondary antibodies applied for $45 \mathrm{~min}$ at room temperature the following day. Nuclei were counterstained with DAPI $(0.1 \mu \mathrm{g} / \mathrm{mL}$; Sigma). Images were acquired on a Zeiss LSM 700 confocal microscope and analyzed with ImageJ software.

\section{Western blotting}

Cells were lysed in RIPA buffer containing phosphatase inhibitor cocktail (Sigma) and protease inhibitor cocktail (Sigma) on ice for $15 \mathrm{~min}$, and protein content was quantified using a Pierce BCA Protein Assay Kit (Thermo Fisher Scientific). Ten micrograms of protein per sample was resolved by electrophoresis and transferred to PVDF membranes. Membranes were blocked for $1 \mathrm{~h}$ at room temperature with $5 \%$ milk in Tris-Buffered Saline containing 0.1\% Tween-20 (TBS-t; Sigma) and incubated overnight with either antiTCOF1 (1:1,000; Abnova) or anti-beta actin (1:10,000; Sigma). Membranes were washed with TBS-t and incubated with horseradish peroxidase (HRP)-conjugated secondary antibodies for $1 \mathrm{~h}$ at room temperature. Membranes were washed and developed using the Pierce ECL2 western blotting substrate (Thermo Fisher Scientific).

\section{Migration assays}

NC and MSC were plated onto six-well plates and allowed to form a confluent monolayer. The cell monolayer was then scratched in a straight line to make a "scratch wound" with a 1-mL pipette tip, and the cell debris was removed by washing the cells with phosphate-buffered saline (PBS). Cells were maintained in FSB (for NC cells) or Dulbecco's modified Eagle's medium (DMEM) $+10 \%$ FBS (for MSC), and images of the closure of the scratch were captured at different time points as indicated.

A chemotaxis assay was performed using the CytoSelect ${ }^{\mathrm{TM}}$ 24-Well Cell Migration and Invasion Assay Combo Kit (Cell Biolabs) following the manufacturer's instructions. Briefly, $5 \times 10^{5}$ cells were plated in a prewarmed 24-well migration plate in FSB media. The chemoattractant FGF8B $(30 \mathrm{ng} / \mathrm{mL}$; R\&D Biosystems) was added separately to FSB media. Cell media was added to the lower well of the migration plate. FSB media was used as a control. Cells were incubated for $8 \mathrm{~h}$ at $37^{\circ} \mathrm{C}$, and quantification was performed following the manufacturer's instructions.

For single cell analysis of cell migration, cells were imaged real time on an IN Cell Analyzer 2200 (GE Healthcare Life Sciences) with images collected every $30 \mathrm{~min}$ for a 12 -h period. Cells were tracked using the Pointing Cell Tracking plugin for ImageJ (https://imagej.nih.gov/ij/plugins/pointing-cell-tracking/ index.html), and migratory profiles were generated using the freely available Chemotaxis and Migration Tool from Ibidi (ibidi.com/chemotaxis-analysis/171-chemotaxis-and-migrationtool.html).

\section{Microarray hybridization and analysis}

RNAs isolated from H9s HESC, neuroectoderm [34], and NC passage 2 and 7 (NC P2, NC P7) were hybridized with Illumina Human HT-12 BeadChip (Illumina, Inc., San Diego, CA; www.illumina.com). All the data processing and analysis were performed using the algorithms included with the Bioconductor package beadarray and Lumi implemented in $\mathrm{R}$ software environment for statistical computing and graphics (R Foundation for Statistical Computing, Vienna, Austria; www.r-project.org).

\section{Microinjection of HPSC-derived NC cells and HPSC-derived endoderm cells in chicken embryos}

For injections into the cardiac NC premigratory region, chicken (Gallus domesticus) eggs (Winter Egg Farm, Cambridge, United Kingdom) were incubated in a digital cabinet incubator (OVA Easy 380; Brinsea) for $32 \mathrm{~h}$ until Hamburger and Hamilton stage 9-10 (HH9-10). Eggs were windowed and injected under the embryo with India Ink to improve contrast. Small cuts were made with a BD Microlance needle (size 3) through the vitelline membrane and ectoderm directly adjacent to the neural tube, at a level just posterior to the forming otic vesicle. Clumps of 50-100 cells in Matrigel ${ }^{\mathrm{TM}}$ were injected into the cut site using a pulled glass capillary tube. Eggs were resealed with tape and cultured a further 20, 42, and $108 \mathrm{~h}$ after injection to visualize migrating NC cells at HH16, $\mathrm{HH} 17$, and $\mathrm{HH} 26$, respectively. HPSC-derived endoderm cells were used as a negative control and generated as previously reported [35].

For systemic injections into developing chicken embryo, eggs were incubated until $\mathrm{HH} 24$. A small window was made, and 500-1,000 NC cells were administered into the extraembryonic vessels. Either $\mathrm{GFP}^{+} \mathrm{HPSC}$-derived NC cells or $\mathrm{GFP}^{+}$HPSC-derived endoderm cells were administered as previously reported $[16,36]$. The window was covered with parafilm (VWR), and eggs were placed horizontally in the incubator until HH34. Embryos were stained using whole mount immunocytochemistry as previously described [36].

\section{Differentiation of the NC to various cell types}

NC populations were differentiated to SMC using a combination of PDGF-BB (10 ng/mL; Peprotech) and TGF- $\beta 1$ ( $2 \mathrm{ng} / \mathrm{mL}$; Peprotech), as previously reported [37]. NC differentiation to neuronal populations and MSC was performed as 
previously reported [21]. NC differentiation to melanocytes was performed as previously described [38]. MSC populations were subsequently differentiated to chondrocytes, adipocytes, and osteocytes using the StemPro Chondrogenesis Differentiation Kit (Thermo Fisher Scientific), StemPro Adipogenesis Differentiation Kit (Thermo Fisher Scientific), and StemPro Osteogenesis Differentiation Kit (Thermo Fisher Scientific), respectively, following the manufacturer's instructions.

\section{Cell proliferation assay}

To assess cell proliferation, the CellTiter 96 NonRadioactive Cell Proliferation Assay [[3-(4, 5-dimethylthiazolyl2)-2, 5-diphenyltetrazolium bromide] (MTT); Promega] Kit was used, as per the manufacturer's instructions.

\section{Freezing and thawing of NC cells}

Confluent NC cells were dissociated with TrypLE Express (Thermo Fisher Scientific). NC cells were pelleted and resuspended in CDM-PVA media. $2 \times 10^{6}$ cells were added to a cryovial using $90 \%$ CDM-PVA media with $10 \%$ dimethyl sulfoxide (DMSO). NC cryovials were thawed at $37^{\circ} \mathrm{C}$, and cells transferred to a $15 \mathrm{~mL}$ Falcon tube containing $8 \mathrm{~mL}$ of fresh CDM-PVA media. Cells were centrifuged for $3 \mathrm{~min}$ at $1,200 \mathrm{rpm}$. Cell pellets were resuspended in $1 \mathrm{~mL}$ FSB media, and $3 \times 10^{5} \mathrm{NC}$ cells were plated per well of a $0.1 \%$ gelatin-coated well of a six well plate. Cells were incubated at $37^{\circ} \mathrm{C}$ overnight in a $5 \% \mathrm{CO}_{2}$ incubator. Cell viability was assessed as previously reported [39].

\section{Small interfering RNA knockdown and transient transfections}

TCOF1 messenger RNA (mRNA) was knocked down with small interfering RNA (siRNA; Thermo Fisher Scientific Assay ID S13920). siRNA transfection (25 nM) was performed using DharmaFECT-1 transfection reagent (Dharmacon) following the manufacturer's instructions.

\section{Generation of a TCOF1-targeting CRISPR guide RNA/Cas9 construct}

A guide RNA (gRNA) targeting the TCOF1 gene was designed to target Exon1 according to the rule of $5^{\prime}$ GN20NGG-3' (sequence 5'-TGGCTATGTGCGTGCGGC GC-3'). Oligonucleotides were synthesized and ligated into pSpCas9(BB)-2A-Puro (PX459) V2.0 as previously reported [40].

\section{Gene targeting}

For gene targeting, $2.5 \times 10^{6}$ HIPSC were electroporated with $1 \mu \mathrm{g}$ of generated TCOF1 targeting Cas9 plasmid in $100 \mu \mathrm{L}$ of nucleofection mix from the P3 Primary Cell 4DNucleofector X Kit (Lonza) using a 4D-nucleofector system (Lonza). Transfected cells were plated onto DR4 strain feeders (Jackson Laboratory) and cultured in advanced DMEM/F12 (Gibco) +20\% KOSR supplemented with FGF2 ( $4 \mathrm{ng} / \mathrm{mL})$ and Rho Kinase inhibitor (Y-27632, $10 \mu \mathrm{M})$. One day after transfection, cells were selected with puromycin ( $1 \mu \mathrm{g} / \mathrm{mL}$; Sigma) for $36 \mathrm{~h}$. Resistant colonies were picked and expanded, and mutation introduction was assessed by PCR and Sanger sequencing.

\section{Statistics}

One-way analysis of variance (Tukey's multiple comparison test) and two-sided Student's $t$-test were used to determine statistically significant differences between the groups. Results are presented as mean \pm standard error of the mean. $P$ values $\leq 0.05$ were considered statistically significant. All experiments represent the results of at least three independent biological replicates (measurements of biologically distinct samples). $* P<0.05$; $* * P<0.01$; $* * * P<0.001$.

\section{Results \\ Dissociation of differentiating neuroectoderm during early development promotes NC differentiation}

It has been demonstrated that neuroectoderm can be generated from HPSC utilizing a combination of FGF2 and the TGF- $\beta$ inhibitor SB-431542 (referred to herein as FSB) for 7 days [16]. Our group has previously reported the expression of some NC markers during this differentiation process, including $S N A I 1, S N A I 2$, and $P A X 3$, suggesting that a mixed cell population may be generated at these stages [37].

We hypothesized that cell dissociation with trypsin, a serine protease, might facilitate EMT and the generation of the NC from this population [41-45]. We, therefore, differentiated the H9s HESC line to neuroectoderm over 7 days with FSB media, with and without WNT3A $(25 \mathrm{ng} / \mathrm{mL}$; R\&D Systems).

At day 7 of differentiation, the neuroectoderm was trypsinized and replated as single cells in CDM-PVA media supplemented with FGF2 and SB-431542 (FSB) \pm WNT3A for up to five passages. qRT-PCR over this period showed increased expression of NC differentiation-associated genes (PAX3, ZIC1, CD49, and SOX9) and the mesenchymal gene (VIM) (Fig. 1A). Conversely, neuroectoderm genes (OLIG3 and $P A X 6)$ and an epithelial gene $(C D H 1)$ were downregulated (Fig. 1A), suggesting that cell dissociation promoted NC marker expression and EMT. Interestingly, the addition of WNT3A did not have any effect on the expression of these markers during the differentiation process (Fig. 1A).

To further analyze the onset of NC markers during the differentiation of HESC to neuroectoderm over the first 7 days, we assessed the expression of $\mathrm{NC}(H N K 1, P 75)$ and neuroectoderm (PAX6) genes on a daily basis. We observed the greatest expression of $H N K 1$ and $P 75$ on day 4 of the differentiation process (Supplementary Fig. S1A). Therefore, we hypothesized that dissociation of the developing neuroectoderm at day 4 instead of day 7 may increase the efficiency of $\mathrm{NC}$ generation during the differentiation process.

We analyzed SOX1 and P75 expression by flow cytometry in passaged and nonpassaged neuroectoderm at days 4 or 7 of differentiation in FSB. SOX1 is the earliest known specific marker of the neuroectoderm lineage and is activated during gastrulation [46]. Neuroectoderm passaged at day 4 (D4 P1) demonstrated a higher induction of P75 and lower expression of SOX1 compared with nonpassaged cells or cells split at day 7 (Fig. 1B). Furthermore, the cells split at day 4 attached and survived better than the cells split at day 7 (data not shown). 
Consistent with these results, TFAP2A, an essential transcription factor for the development of the NC [47,48], was highly expressed in the D4 P1 population (Fig. 1C). SOX1 was detected at the edges of the neuroectoderm at days 4 and 7 of differentiation in FSB by immunocytochemistry (Fig. 1D).
Following passage at day 4, SOX1 expression was significantly reduced. Conversely, SOX1 positive cells remained if passaging was delayed until day 7 (Fig. 1D), suggesting that the greatest reduction of SOX1 was obtained by passaging the differentiating neuroectoderm in FSB on day 4. Furthermore,
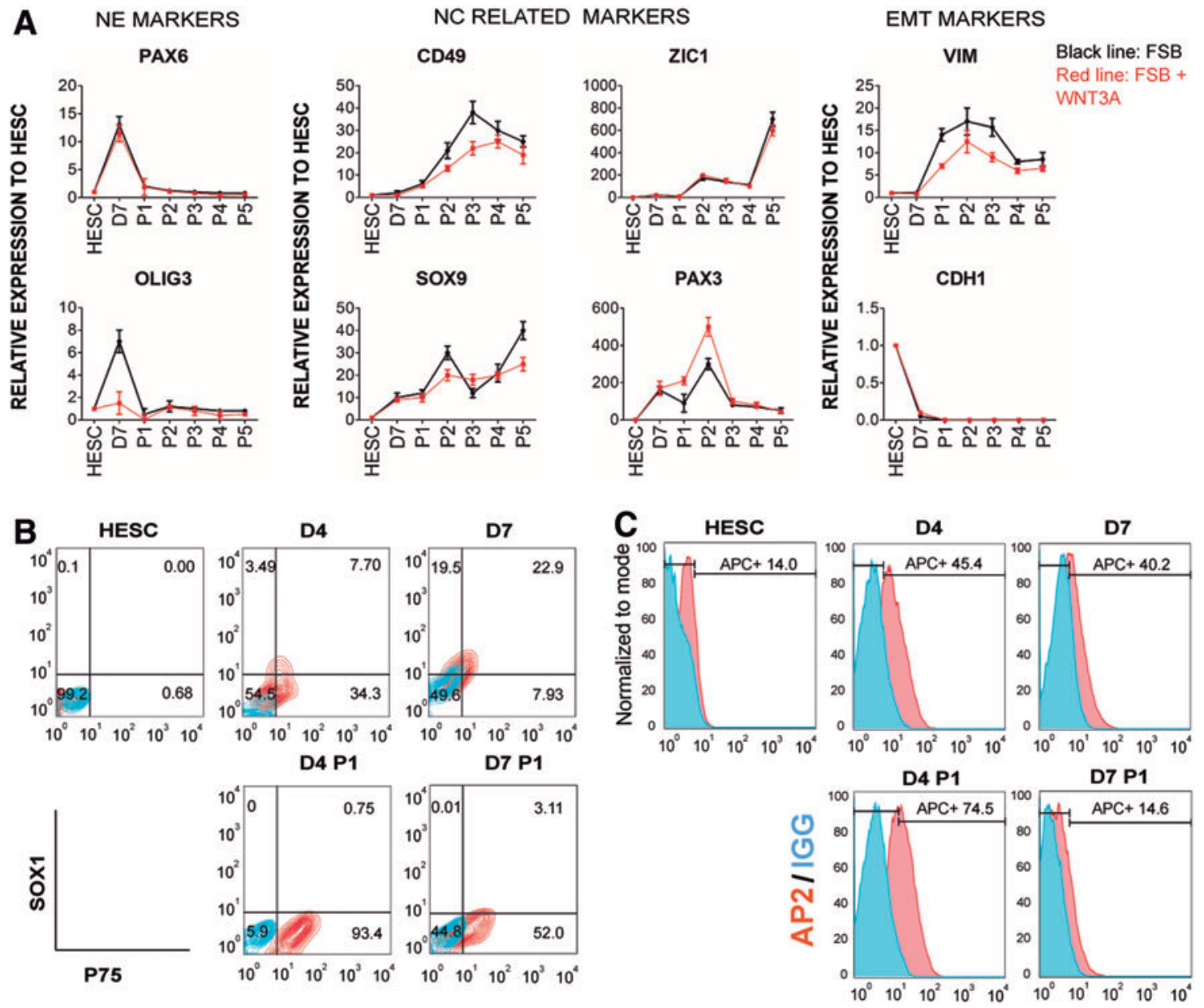

FIG. 1. Differentiation of NC cells from HESC. (A) Expression of NE, NC, and EMT specific genes by qRT-PCR during H9s HESC differentiation in CDM, supplemented with FGF2, SB-431542 (FSB) and with (red line) or without WNT3A (black line). Cells were studied as undifferentiated HESC, NE day 7 (D7), and with serial passages (P1-P5). mRNA levels were normalized to the housekeeping gene $P B G D$ and then presented relative to HESC expression which was set to 1 . The results presented are representative of three independent experiments. (B) Flow cytometry analysis of NE marker SOX1 and NC marker P75 in the differentiation of H9s HESC to NE. FSB media was used to differentiate H9s HESC to NE over 4 or 7 days. SOX1 and P75 were analyzed before (D4, D7) and after (D4 P1 and D7 P1) splitting the NE at indicated time points. Red contour plots represent SOX1+P75 double stained populations, and blue contour plots represent IgG control staining. (C) Histogram of flow cytometric analysis of the NC marker TFAP2A (AP2) in H9s HESC differentiation with FSB to NE over 4 or 7 days. TFAP2A expression was measured before (D4, D7) and after (D4 P1, D7 P1) splitting the NE at indicated time points. Red histograms represent TFAP2A stained populations, and blue histograms represent IgG control staining. (D) Immunocytochemistry for the NC marker HNK1 and the NE marker SOX1 with DAPI counterstain in H9s HESC differentiation to NE. Upper panels, differentiation of HESC colonies over 4 days (D4) and after passage (D4 P1). Bottom panels, differentiation over 7 days. Colonies passaged at day 7 showed a remaining SOX1+ population (D7 P1, white square) shown in D7 P1 ZOOM. The SOX1+ population was not detected in colonies passaged on day 4. White scale bar: $100 \mu \mathrm{m}$, blue scale bar: $50 \mu \mathrm{m}$, red scale bar: $20 \mu \mathrm{m}$. (E) Expression of the NC marker TFAP2A and NE marker GBX2 by qRT-PCR. H9s HESC colonies were differentiated with FSB media over 4 or 7 days to NE. RNA samples were collected at days 4 and 7 before and after passage (D4, D7, D4 P1, and D7 P1, respectively). The relative mRNA level was normalized to the housekeeping gene $P B G D$. The results are presented as mean \pm SD of three independent experiments. $* P<0.05$; **P $P<0.01$, two-sided Student's $t$-test. CDM, chemically defined media; EMT, epithelial-mesenchymal transition; FGF, fibroblast growth factor; HESC, human embryonic stem cell; mRNA, messenger RNA; NC, neural crest; NE, neuroectoderm; PBGD, porphobilinogen deaminase; qRT-PCR, quantitative real-time polymerase chain reaction; SD, standard deviation. Color images available online at www.liebertpub.com/scd 

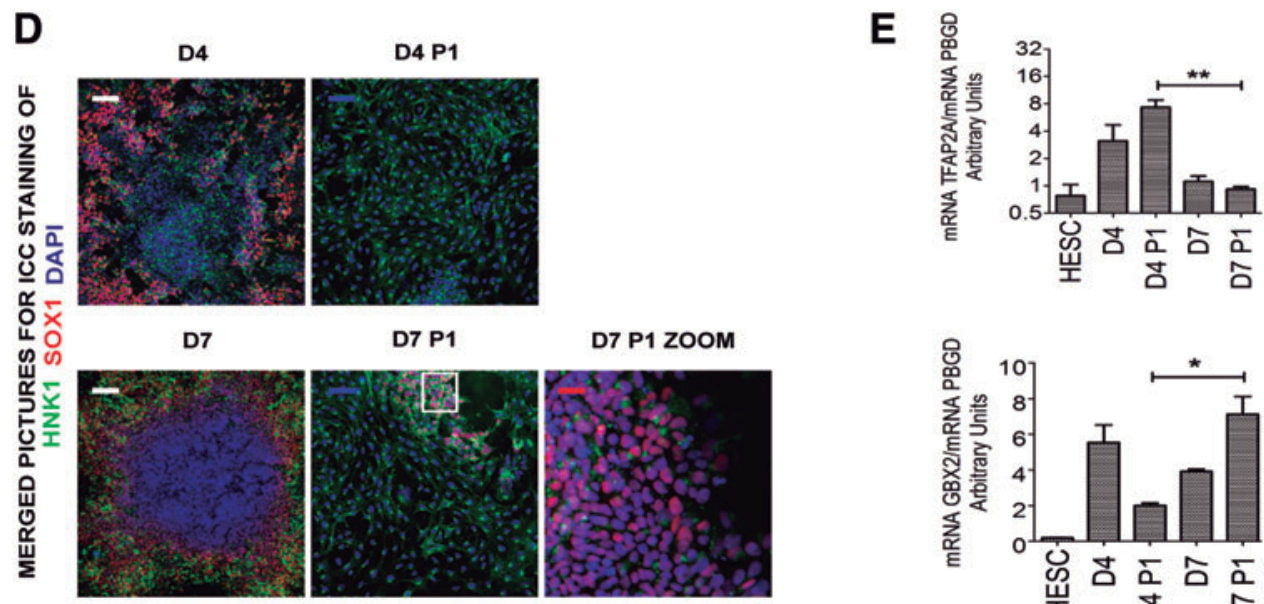

FIG. 1. (Continued).

HNK1 was highly expressed in passaged neuroectoderm at day 4 (Fig. 1D), and qRT-PCR analyses confirmed that these cells showed significantly higher levels of NC marker TFAP $2 A$ and lower levels of neuroectoderm marker $G B X 2$ [49] than cells from day 7 passaged neuroectoderm (Fig. 1E).

Together, these findings suggest that differentiation of neuroectoderm from HPSC can generate a mixed population containing both neuroectoderm and NC cells. The NC population can be enriched from the differentiating neuroectoderm by passaging at day 4 of the protocol.

\section{Serial passage increases purity of NC cells}

Following passaging at day 4, cells were maintained in FSB medium upon reaching confluence (NC P1). Every 4 days, the confluent NC cells were split at a 1:3 ratio and seeded for the next passage (Supplementary Fig. S1B). After two passages cells expressed the NC proteins SOX9, HNK1, and P75 and did not express the neuroectoderm marker SOX1 (Fig. 2A). We detected expression of the NC markers P75 and TFAP2A by flow cytometry following subsequent passaging (Fig. 2B, C) [21]. While over $80 \%$ of the early NC population expressed P75, only $50 \%$ of the population was double positive for both P75 and TFAP2A at this stage (Fig. 2C). Furthermore, SOX10, a marker of migratory NC, was induced at D4 (Fig. 2D). These data confirmed that we were generating $\mathrm{NC}$ at this time point of the differentiation process, although the yield was suboptimal.

We questioned whether the differentiated $\mathrm{NC}$ would retain their NC-like identity following further expansion. Interestingly, the expression levels of the $\mathrm{NC}$ markers $P A X 3$ and ZIC1, markers of early NC induction [50,51], peaked at passage $3(\mathrm{P} 3)$. In contrast, $S O X 9$, which is an essential factor for the further development of migratory NC [52], was observed to be highly expressed at passage 8 (P8) (Fig. 2E, top). The expression levels of the NC markers P75, TFAP2A, and TWIST1 increased significantly upon serial passaging (P1, P7, and P13), as assessed by qRT-PCR (Fig. 2E, bottom), suggesting that this approach could purify the NC population.

FIG. 2. Increased purity and maturation of HESC derived NC cells with serial passages. (A) Immunostaining of the NC markers SOX9, P75, and HNK1 and the NE marker SOX1 with DAPI counterstain, in H9s HESC-derived NC after two passages (NC P2) in FSB media. Blue scale bar: $50 \mu \mathrm{m}$, white scale bar: $100 \mu \mathrm{m}$. (B) Flow cytometric analysis of P75 and HNK1 in H9s HESC after 4 days of differentiation in FSB media before passage (H9s 4D IN FSB) and H9s-derived NC after three passages maintained in FSB as single cells (H9s NC P3). Red contour plots represent HNK1+P75 double stained populations, blue contour plots represent IgG controls. (C) Flow cytometric analysis of TFAP2A and P75 in the differentiation of undifferentiated H9s and H9s-derived NC after three passages maintained in FSB as single cells (H9s NC P3). Colonies were passaged at day 4 of differentiation. Red contour plots represent TFAP2A+P75 double stained populations, blue contour plots represent IgG controls. (D) Flow cytometric analysis of SOX17 and SOX10 in H9s HESC, endoderm cells differentiated from H9s (H9s END), and H9s HESC at day 4 of differentiation in FSB without splitting (H9s 4D IN FSB). SOX10 induction was observed following 4 days of FSB treatment, whereas the population was negative for expression of the endoderm marker SOX17. Red contour plots represent SOX17+SOX10 double stained populations, and blue contour plots represent IgG controls. (E) Top panel: qRT-PCR expression analysis of NC markers PAX3, ZIC1, and SOX9. Bottom panel: qRT-PCR expression analysis of NC markers P75, TFAP2A, and TWIST1. mRNA of HESC-derived NC cells at different passages was used to perform the qRT-PCR. The relative mRNA levels were relative to NC passage 1 levels (NC P1). The relative mRNA level was normalized to the housekeeping gene PBGD. P1, P3, P5, P7, P8, and P13: number of passages of the NC cells as single cells. Results presented as mean $\pm \mathrm{SD}$ of three independent experiments. $* P<0.05$; $* * P<0.01 ; * * * P<0.001$. Twosided Student's $t$-test. (F) Representative images of in vitro scratch wound assay performed in H9s HESC derived NC cells (NC P7). Pictures were obtained from 0 to $24 \mathrm{~h}$ postscratch. White scale bar: $100 \mu \mathrm{m}$. (G) Flow cytometric analysis of TFAP2A and P75 in H9s HESC and H9s HESC-derived NC passage 12 in FSB (NC P12). Red contour plots represent TFAP2A+P75 double stained populations, and blue contour plots represent IgG controls. (H) Chemotaxis potential and migration in representative H9s-derived NC P7 was assessed using a CytoSelect ${ }^{\mathrm{TM}}$ Transwell Cell Migration Assay Kit. The NC chemoattractant FGF8B was added to FSB media to assess migration. DMEM/F12+10\% fetal bovine serum (SERUM) was used as a positive control of migration. FSB migration without any chemoattractant was set as 1, and data are expressed relative to this. Equal cell numbers were used in each condition, and results are presented as mean $\pm \mathrm{SD}$ of three independent experiments. $* P<0.05$; $* * P<0.01$. DMEM, Dulbecco's modified Eagle's medium. Color images available online at www.liebertpub.com/scd 
To further confirm that the NC cells retain their NC identity and show purification with passaging, we used flow cytometry to examine the expression of $\mathrm{NC}$ and non-NC markers. Furthermore, we repeated the flow analysis of P75 and TFAP2A in late passages of NC (P12) and observed that 99\% of the cells expressed P75 and 69\% showed both P75 and TFAP2A (Fig. 2G). Similar results were also obtained using HIPSC to generate NC cells (Supplementary Fig. S2A-C).

To assess if the purified NC population contained additional cell populations, we measured the expression of endoderm (SOX17 and EOMES), mesoderm, (NKX2.5 and $K D R$ ), and neuronal (TUBB3 and MAP2) genes in the populations (Supplementary Fig. S2E). We observed negligible

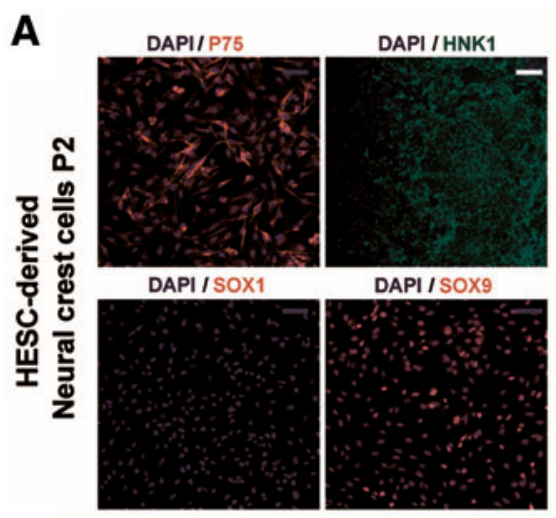

B

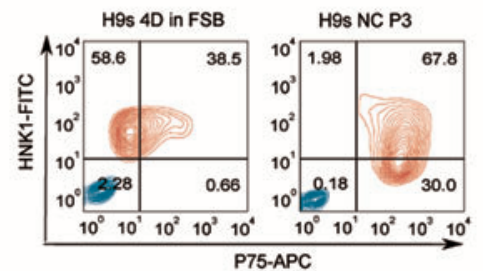

C
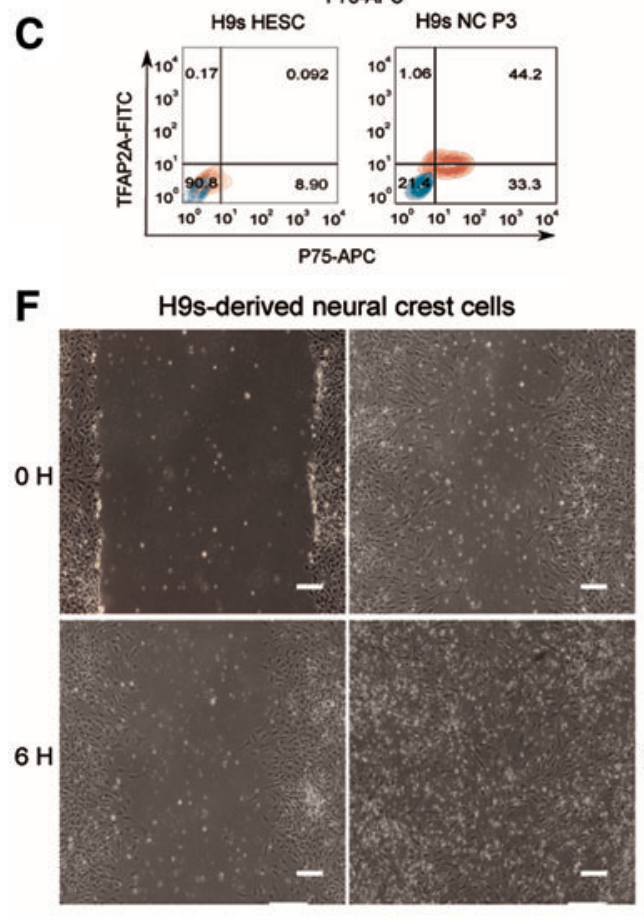

expression of these genes in the $\mathrm{NC}$ populations compared to HPSC-derived endoderm, mesoderm, or neurons, demonstrating that the NC did not contain a mixture of these populations in culture. Taken together, these data suggest that $\mathrm{NC}$ purification may be achieved by serial passaging of NC cells in FSB media. Furthermore, we found that we could expand the NC cells up to passage 15 without losing their NC identity [53].

\section{NC cells are proliferative and migrate in response to specific chemoattractants}

To assess whether passage number or cell density affects the proliferation rate of the differentiated $\mathrm{NC}$, we performed sequential cell counting and an MTT assay at different NC
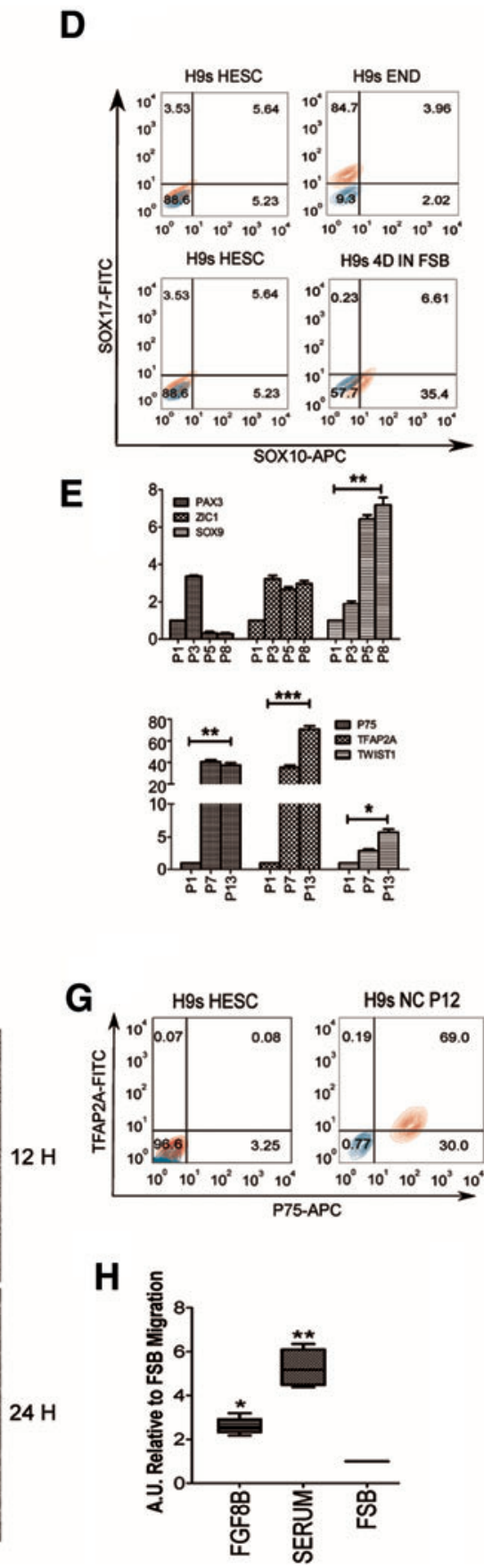
passages (Supplementary Fig. S2C, D). The MTT Cell Proliferation Assay is used to calculate the cell proliferation rate. The yellow tetrazolium MTT is reduced by active cells to purple formazan that can be solubilized and quantified by spectrophotometric means.

We found that the proliferation rate of $\mathrm{NC}$ was independent of passage number (Supplementary Fig. S2C) or cell density (Supplementary Fig. S2D). In vivo, NC cells migrate over great distances in response to chemotactic cues to contribute to tissue development during embryogenesis [54]. To validate a similar migratory function in our cells, we performed an in vitro scratch assay. NC cells were able to migrate and cover the scratch within $24 \mathrm{~h}$ (Fig. 2F). FGF8 is chemotactic and chemokinetic for $\mathrm{NC}$ in vivo and in vitro $[19,54]$. We, therefore, examined whether the HESC-derived NC cells would respond to this cue using the CytoSelect 24well Cell Migration Assay $(8 \mu \mathrm{m})$. In response to FGF8B, NC migration was significantly greater than in FSB media alone (Fig. 2H). Overall, these data demonstrate that the HESC-NC cells recapitulate critical $\mathrm{NC}$ functions.

\section{Early passages of HESC-NC comprise a mixed population of neural progenitors and premigratory NC cells that is purified with serial passage}

To uncover the differences between early and later passages of NC, we performed a microarray gene expression analysis using H9s HESC and three independent differentiations of H9s HESC to neuroectoderm [34], NC at passage 2 (P2), and NC at passage 7 (P7). Hierarchical clustering separated these populations (Fig. 3A). Interestingly, PCA analysis distributed NC P2 in between neuroectoderm and NC P7 population (Fig. 3B). Based on this, we hypothesized that the early $\mathrm{NC}$ population could be a mixture of neuroectoderm and $\mathrm{NC}$ cells.

The NC P2 population expressed markers of neural progenitors, including AXIN2 [55], FGFR3 [56], and NOTCH3 [57] (Fig. 3C). These genes, however, were markedly downregulated at later passages (Fig. 3C). Furthermore, the NC P2 population expressed markers of neural plate border/premigratory NC cells, including $C D H 2$ [58], $C D H 6$ [59], FOXD3 [60,61], BMP7, and RHOB [9,62] (Fig. 3C). These neural plate border genes are thought to promote conversion of premigratory $\mathrm{NC} \mathrm{P} 2$ to migratory cells as they also expressed migratory markers such as B3GAT1 (HNK1) $[63,64]$ in this mixed population (Fig. 3C).

As the WNT signaling pathway has been identified as playing important roles in NC development in vivo [23,6567], we wondered why exogenous WNT3A was not required in our in vitro differentiation system. Interestingly, genes related to activation of WNT signaling, such as WNT1, FZD3, DVL2 [68], and MSX1 [69], were significantly expressed in the early NC P2 population (Fig. 3C). These data suggest that there may be endogenous activation of WNT during the early stages of NC differentiation, as observed in the neural plate border intermediate cells in vivo [69] and in vitro [23].

Interestingly, it has been proposed that $\mathrm{NC}$ induction requires intermediate levels of BMP signaling [70], as part of a BMP gradient between epidermal ectoderm which expresses both BMP7 and BMP4 [71] and the neural plate. BMP7 induces the expression of the RHOB protein in the cells destined to become $\mathrm{NC}$ in the neural plate border
[9,62]. Consistent with developmental NC induction, $B M P 7$ and $R H O B$ were highly upregulated in the early NC P2 population compared with NC P7. In contrast, the NC P7 population expressed higher levels of migratory $\mathrm{NC}$ genes such as SOX10, SOX9, TWIST1, and P75 [52,58,72,73] compared to the early NC P2 population (Fig. 3C).

Finally, we plotted gene expression differences in the NC pathway using an open source pathway archive (WikiPathway WP2064 revision 47071). Most genes upregulated at NC P2 corresponded to genes expressed in premigratory NC cells (Fig. 3D). This population also showed some migratory $\mathrm{NC}$ genes, suggesting a mixed population. However, genes upregulated in the NC P7 population corresponded for the most part with genes expressed in the migratory NC (Fig. 3D). These data revealed evidence of endogenous WNT and BMP activity in the NC P2 population. In these early passages, a mixed NC population could be purified in vitro after several further passages to express a migratory $\mathrm{NC}$ transcriptional profile by passage 7 .

\section{HESC-derived NC cells survive, engraft, migrate, and differentiate in vivo at NC specific location within the ascending aorta and the brain}

NC cells should engraft in the appropriate locations for $\mathrm{NC}$ derivatives. In avian embryos, the fate of the NC is well established. The removal of the dorsal neural tube between the otic vesicle and the third somite in chicken embryos results in a variety of defects of the derivatives of the arch arteries [74]. Chicken embryos have previously been used as a host for studying the differentiation potential of human stem cells $[36,75,76]$. To demonstrate that the NC cells have the potential to migrate and differentiate to their specific locations in vivo and that freeze/thaw cycles do not affect the potential of the NC cells, we performed several experiments engrafting or injecting $\mathrm{NC} \mathrm{GFP}^{+}$cells into chicken developing embryos.

Fluorescent $\mathrm{GFP}^{+}$HESC-derived NC P1 cells were thawed, harvested, and passed to NC P2 cells. Those cells were split, counted, and embedded into Matrigel for chicken embryo engraftment. Clumps of 50-100 cells were engrafted between the otic vesicle and the third somite, adjacent to the neural tube at HH9-10. Some embryos were harvested 20 and $42 \mathrm{~h}$ postengraftment (HH16-17) to monitor the success of the engraftment. Observation under an inverted epifluorescent microscope showed that they exhibited a lump of $\mathrm{GFP}^{+}$cells in the region of the otic vesicle. Furthermore, migrating $\mathrm{GFP}^{+}$ cells were detected in all the embryos (Fig. 4A, B).

Four and a half days postengraftment (HH29), wholemount confocal immunofluorescence imaging of the developing heart and ascending aorta was performed. A significant number of $\mathrm{GFP}^{+}$cells were found in the ascending aorta expressing ACTA2 (Fig. 4C). Furthermore, $\mathrm{GFP}^{+}$cells were not found in the aorta following engraftment of $\mathrm{GFP}^{+}$ HESC-derived endoderm embedded into Matrigel. In addition, we could not detect any NC-derived $\mathrm{GFP}^{+}$cells in the epicardium or myocardium (Fig. 4C). Taken together, these data demonstrate that the engrafted human NC cells can migrate into the ascending aorta and contribute to the SMC population within the chicken embryo.

We also utilized an alternative method that we have previously validated for using chicken embryos as a host to 
A
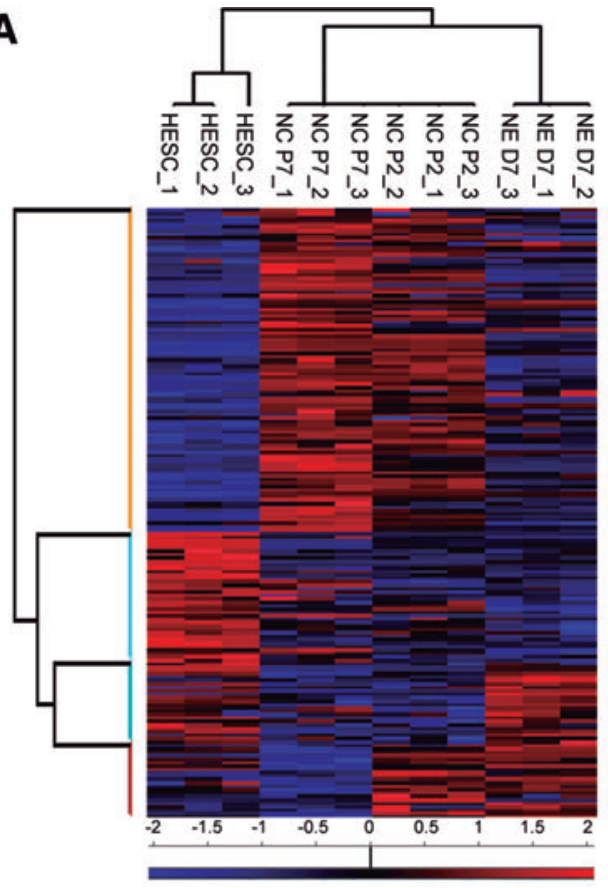

B

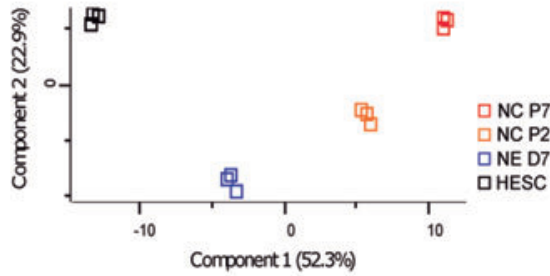

C
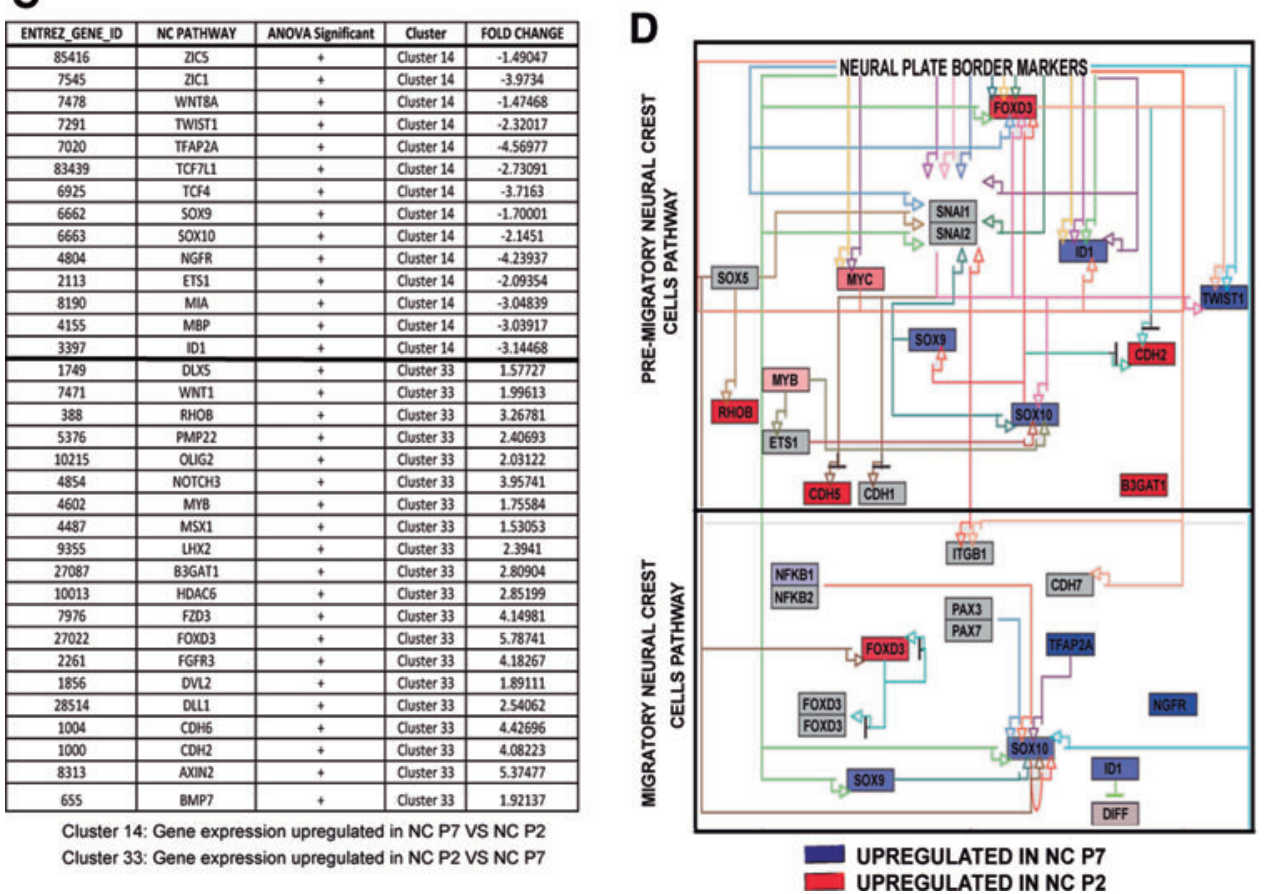

FIG. 3. NC P2 cells are a mix of premigratory $\mathrm{NC}$ and neural progenitor cells that are purified into migratory $\mathrm{NC}$ cells with serial passages (P7). (A) Heat map showing the Euclidean distance metric among HESC, NC P2, NC P7, and NE D7 populations. The global gene expression patterns were sorted based on similarity by hierarchical clustering. Red (upregulation) and blue (downregulation) depict differential gene expression from the mean across all samples. NC P2, NC P7: H9s HESC treated 4 days with FSB then split as single cells for two or seven passages, respectively. NE D7: NE cells obtained from H9s HESC after 7 days in FSB. (B) Principal component analysis showed the NC P2 population as an intermediate population between NE and NC P7. (C) Fold change gene expression of NC and neural markers between NC P2 and NC P7 populations. Cluster 14 depicts the NC genes upregulated in NC P7 compared with NC P2. Cluster 33 illustrates the NC genes upregulated in NC P2 compared with NC P7. Benjamini-Hochberg FDR 0.05 was used as a cutoff for ANOVA of differentially expressed genes. (D) Gene interaction pathway for NC cell differentiation (WikiPathway WP2064 revision 47071). Differentially expressed genes are colored in red for upregulated gene expression in NC P2 or blue for upregulated gene expression in NC P7. PathVisio software was used to depict the NC pathway. Legend: Arrows represent stimulatory interaction, while $\mathrm{T}$ lines represent inhibition. The coloration intensity of the box is dependent on the level of differential expression between these two populations. Those which are highest in NC P7 are blue, and those highest in NC P2 are red. ANOVA, analysis of variance; FDR, false discovery rate. Color images available online at www.liebert pub.com/scd 


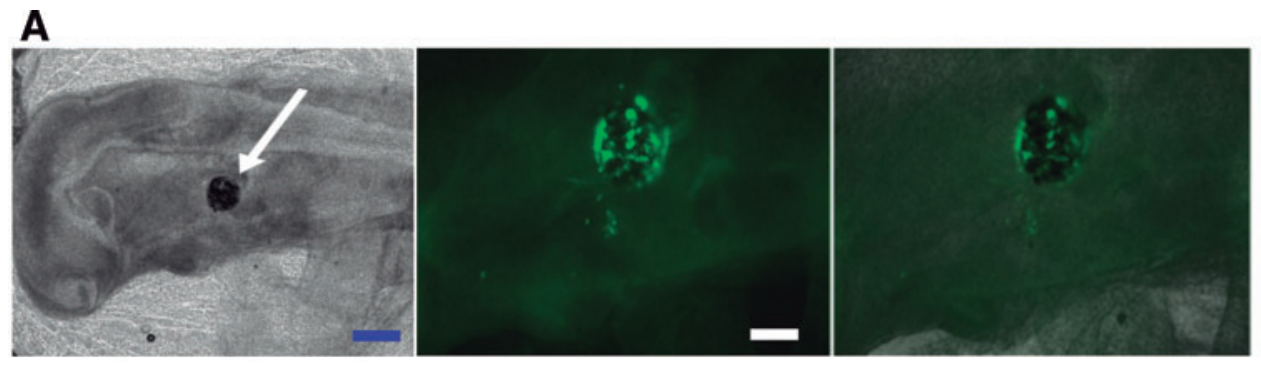

B
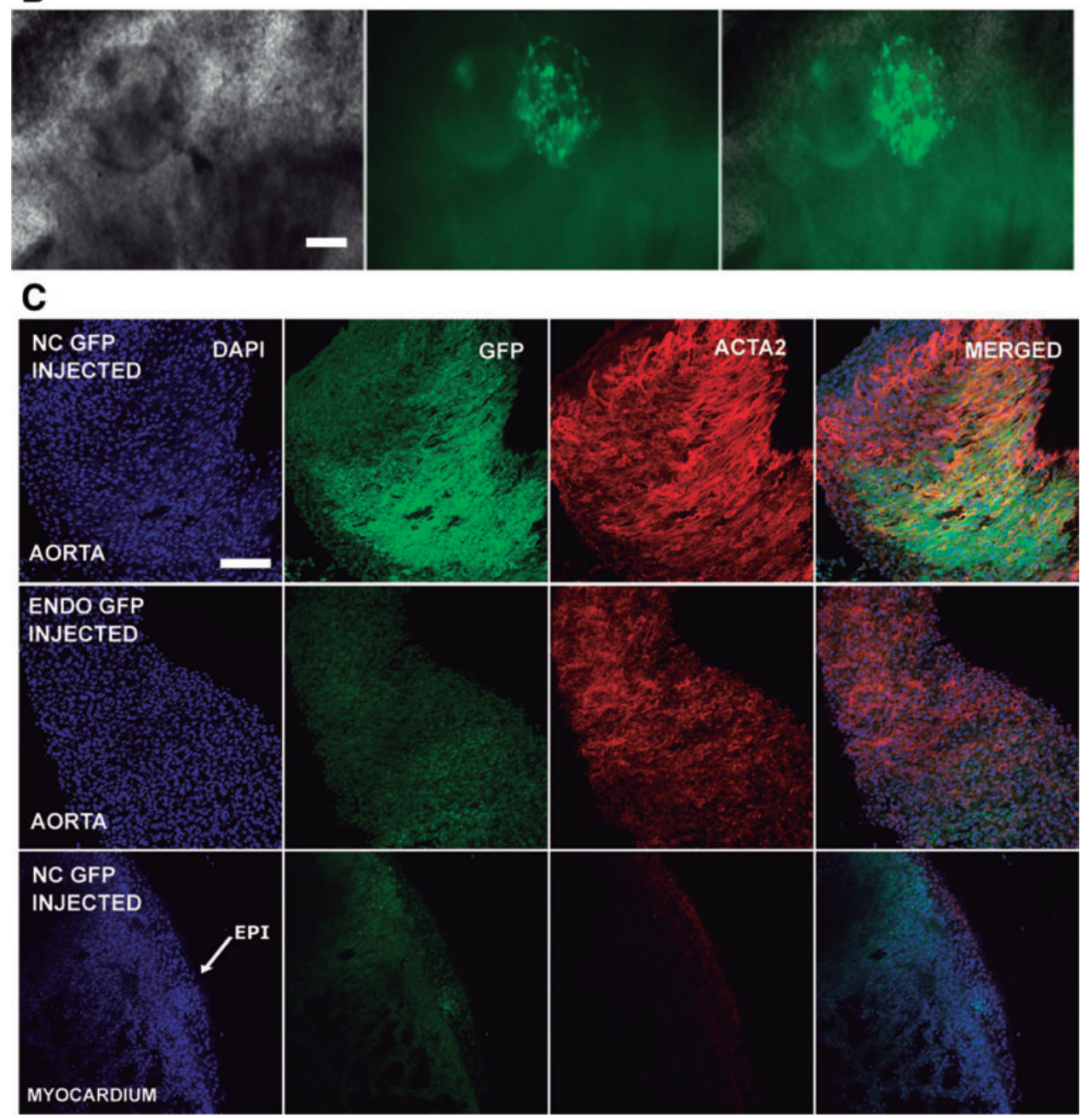

FIG. 4. HESC-derived NC cells engraft, migrate, and differentiate into specific NC developmental location in the chicken embryo. (A) Fluorescent images of HESC-derived NC GFP ${ }^{+}$cells showing survival into the chicken embryo, $20 \mathrm{~h}$ after engraftment at HH16. Clumps of 100-150 HESC-derived $\mathrm{NC} \mathrm{GFP}^{+}$cells at P2 were embedded into Matrigel ${ }^{\mathrm{TM}}$ and engrafted into the chicken embryo at HH9-10. The clumps were inserted in between the otic vesicle and the third somite, near to the neural tube (white arrow, left panel). Blue scale bar: $100 \mu \mathrm{m}$. White scale bar: $50 \mu \mathrm{m}$. (B) Fluorescent images showing HESC-derived NC GFP ${ }^{+}$cells migrating from the Matrigel inside the embryo (HH17) $42 \mathrm{~h}$ after engraftment. White scale bar: $50 \mu \mathrm{m}$. (C) Top row: Confocal images from the chicken embryo aorta 4.5 days after HESC-derived NC GFP ${ }^{+}$cell engraftment (HH29). The $\mathrm{GFP}^{+}$cells migrated to the aortic arch $\left(\mathrm{GFP}^{+}\right.$picture) and differentiated into SMC (ACTA2 picture) contributing to the aortic wall. Middle row: Confocal images from chicken aorta 4.5 days after HESC-derived endoderm $\mathrm{GFP}^{+}$cell insertion $(\mathrm{HH} 29)$. No $\mathrm{GFP}^{+}$cells were visible in the aortic arch of the chicken embryo when HESCderived endoderm $\mathrm{GFP}^{+}$cells were injected instead of the HESC-derived NC GFP ${ }^{+}$cells. Bottom row: Confocal images from chicken myocardium and epicardium 4.5 days after HESC-derived $\mathrm{NC} \mathrm{GFP}^{+}$insertion (HH29). No GFP ${ }^{+}$cells were identified in the epicardium or the myocardium of the chicken embryo. GFP ${ }^{+}$cells were restricted to the aorta. White scale bar: $50 \mu \mathrm{m}$. HH, Hamburger and Hamilton; SMC, smooth muscle cell. Color images available online at www.liebert pub.com/scd 
study the differentiation and integration of HESC-derived cells [36]. $\mathrm{GFP}^{+} \mathrm{NC}$ cells were injected (500-1,000 cells) into the extraembryonic circulation of chicken embryos at $\mathrm{HH} 24$. Embryos were harvested at HH34, and whole-mount confocal immunofluorescence was used for imaging of the brain, ascending aorta, and heart. We detected $10-20 \mathrm{GFP}^{+}$ $\mathrm{NC}$ cells per embryo, around the ascending aorta (Supplementary Fig. S3A-F), and $40-50 \mathrm{GFP}^{+} \mathrm{NC}$ cells per embryo associated with the cerebral cortex vasculature (Supplementary Fig. S3G-J). We could not detect any human NC cells in the subepicardium neither the myocardium (Supplementary Fig. S3K), areas we have previously seen the localization of HESC-derived epicardial cells [36].

HESC-derived NC cells could be clearly discriminated from the host cells by their cell size, high green fluorescence (Supplementary Fig. S3A, D, G), and distinct nuclei (Supplementary Fig. S3B, E, H). Some of the engrafted NC cells around the ascending aorta also expressed ACTA2, suggesting the onset of EMT and SMC differentiation in situ within the ascending aorta (Supplementary Fig. S3C). Together, these results suggest that HESC-derived NC cells have access to ascending aorta in vivo and have the potential to contribute to tissue development in this location.

\section{In vitro differentiation of NC to a variety of cell types}

$\mathrm{NC}$ cells can differentiate in vivo into a wide range of cell types such as neurons, melanocytes [77-79], MSC [80], adipocytes [81], chondrocytes, osteocytes [82,83], and vascular SMC [84]. We, therefore, followed a wide range of established differentiation protocols to confirm that our purified NC cells (NC P7 and further passages) were able to differentiate into their known derivatives.

To assess the capability of the $\mathrm{NC}$ to differentiate into neurons, we plated cells on polyornithine-laminin-coated culture dishes [21]. After 10 days of differentiation, these cells spontaneously differentiated into beta III Tubulin ${ }^{+}$ neurons (Fig. 5A). Neuronal morphology was confirmed by microscopy (Supplementary Fig. S4B). Differentiation to SMC from NC was performed following our previous protocol [34]. SMC proteins such as ACTA2, SM22A, and CNN1 were detected by immunocytochemistry at 12 days of differentiation (Fig. 5B). ACTA2, TAGLN, and other SMC markers such as MYH11, SMTN-B, and MYOCD were also upregulated in differentiated SMC as assessed by qRT-PCR (Supplementary Fig. S4H).

Melanocyte differentiation was achieved following a previously reported protocol [38]. qRT-PCR assessed differentiation for melanocyte markers KIT and MITF [38] and NC markers such as P75 and FOXD3 (Fig. 5C). NC-derived melanocytes showed statistically significant upregulation of KIT and MITF expression and a marked downregulation of $P 75$ and FOXD3. Typical melanocyte morphology was confirmed by microscopy (Supplementary Fig. S4C). MSC were derived from the $\mathrm{NC}$ using a previously reported protocol [21]. Differentiated MSC was positive for CD44 and negative for P75 as assessed by flow cytometry (Fig. 5D) and expressed the MSC marker CD105 (ENG) by qRT-PCR (Supplementary Fig. S4D) [85]. Chondrocytes, adipocytes, and osteocytes were successfully differentiated from NC using specific commercial differentiation media, following the manufacturer's instructions (Thermo Fisher Scientific).
Chondrocyte differentiation was demonstrated by Alcian Blue-positive staining of NC-derived chondrocytes (Fig. 5E). $A C A N$ expression [86] also confirmed chondrocyte lineage (Supplementary Fig. S4E). Adipocyte differentiation was determined by positive oil red $\mathrm{O}$ staining (Fig. 5F) and PPARG [87] expression (Supplementary Fig. S4F). Osteocyte differentiation was shown by positive alizarin red staining (Fig. 5G). COL1A1 [88] and Osteocalcin [89] were detected by immunocytochemistry in osteocytes derived from NC (Supplementary Fig. S4G, top panel). Furthermore, the expression levels of the osteocyte genes sclerostin (SOST) and COL1A1 were measured by qRT-PCR, revealing robust upregulation in NCderived osteocytes (Supplementary Fig. S4G, bottom panel).

Finally, we examined the ability of these cells to function as NC cells after freeze-thawing. NC cells were frozen at passage 7 then defrosted and cultured for two further passages and retained their NC marker expression comparable to passage $9 \mathrm{NC}$ cells that had not undergone freeze-thawing (Supplementary Fig. S4I, top panel). Similarly, freeze-thawing had no effect on the ability of the NC cells to generate derivatives such as SMC (Supplementary Fig. S4I, bottom panel). Furthermore, viability assays performed in thawed NC P2 and NC P7 cells [39] showed a recovery of $89 \% \pm 6.5 \%$ and $92.5 \% \pm 6.25 \%$, respectively, from frozen vials (Supplementary Fig. S4J). The ability to freeze and store these cells is important for the practical utilization of these cells, which can now be bulked up for storage and used as required, without losing NC properties.

\section{Downregulation of Treacle expression by siRNA impairs cell migration in NC and MSC}

Haploinsufficiency of TCOF1 in humans is associated with TCS, a condition characterized by craniofacial abnormalities thought to be due to impaired NC development [90]. To determine whether TCOF1 haploinsufficiency results in NC defects in humans, we initially investigated its role in HPSC-derived NC by siRNA-mediated knockdown. Transfection of TCOF1-targeted siRNA reduced mRNA expression in both NC and MSC as assessed by qRT-PCR (Supplementary Fig. S5A) and at the protein level in the NC by flow cytometry (Supplementary Fig. S5B).

To investigate whether TCOF1 has any role in the differentiation of NC to MSC, we differentiated NC P7 cells transfected with siRNA against TCOF1 or Scramble siRNA to a MSC fate as previously reported [21]. We observed no difference in the capacity for MSC differentiation from TCOF1 knockdown NC with upregulation of CD44 and downregulation of P75 observed in both knockdown and control conditions (Fig. 6A).

In mice, Treacle plays a role in $\mathrm{NC}$ development and proliferation [32]. We assessed if siRNA-mediated knockdown of TCOF1 impaired proliferation of HPSC-derived NC by performing an MTT assay. As in the mouse, we observed decreased proliferation in siRNA-mediated TCOF1 knockdown cells compared to controls (Fig. 6B).

It has previously been suggested that migration is not impaired in $\mathrm{Tcof}^{++-}$mice [32]. To assess the role played by TCOF1 in human NC, we performed a wound healing scratch assay following siRNA-mediated knockdown of TCOF1. We observed impaired migration and scratch closure in TCOF1 knockdown NC compared to controls 

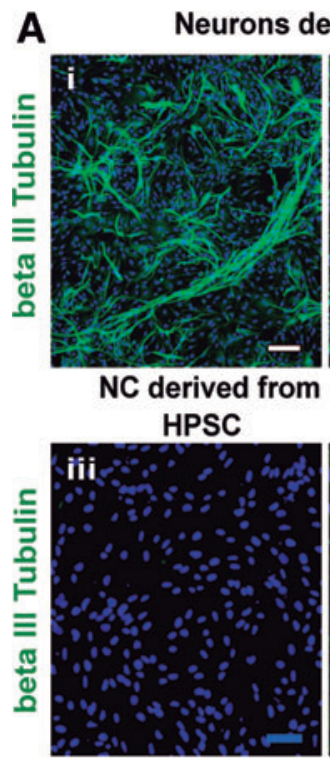

C
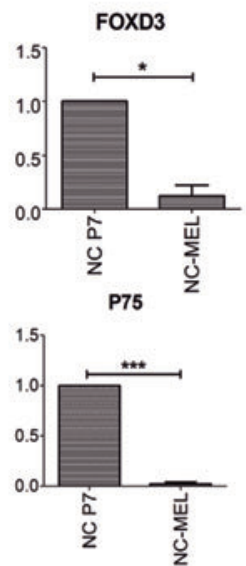

E

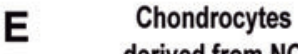
derived from NC

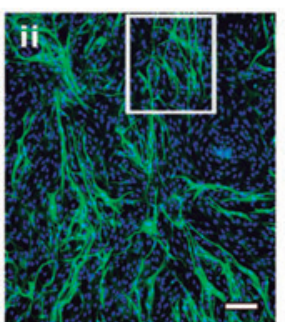

Neurons derived from NC Zoom
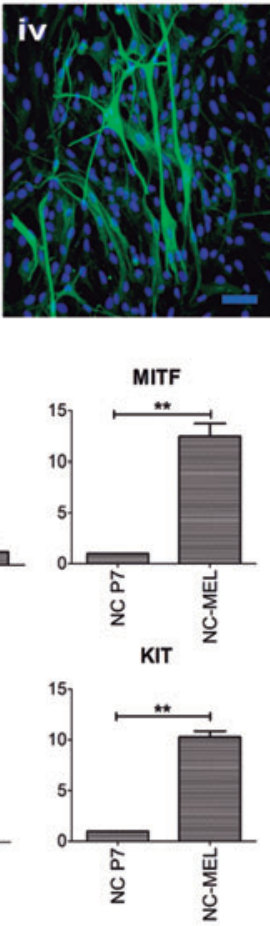

\section{$\mathbf{F}$}

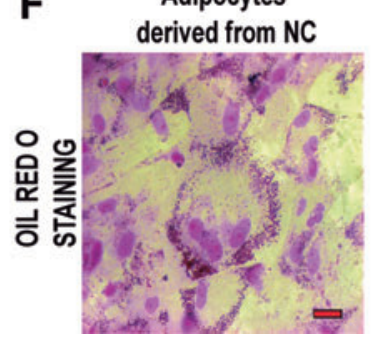

B

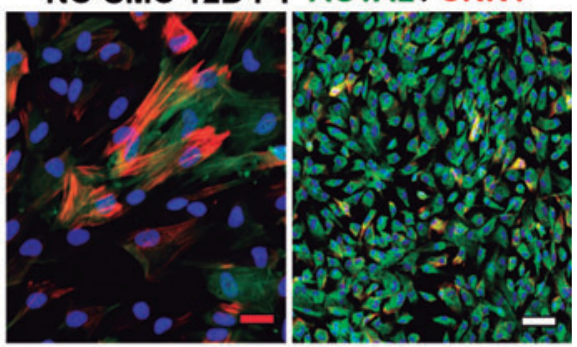

NC-SMC 12D PT SM22A/ CNN1
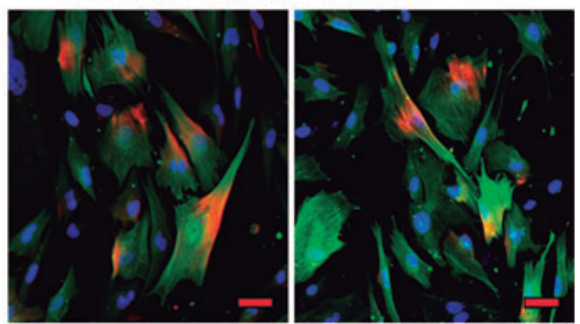

D

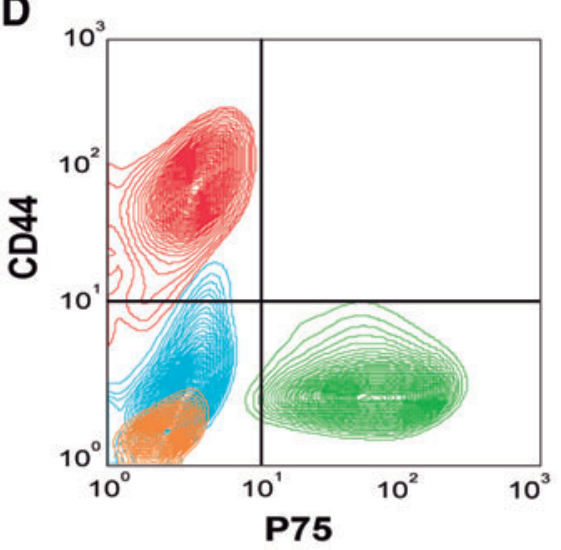

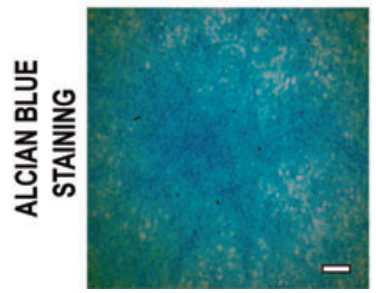

FIG. 5. Differentiation of HESC-derived NC to their derivatives. (A) HESC-derived NC cells were plated on polyornithine-laminin-coated culture dishes to induce neuronal differentiation. (A.i, A.ii) Beta III Tubulin immunocytochemistry showing differentiation to peripheral neurons. (A.iii) NC cells stained with beta III Tubulin as a negative control. (A.iv) Magnification of the white square in (A.ii). White scale bar: $100 \mu \mathrm{m}$, blue scale bar: $50 \mu \mathrm{m}$. Representative images of three independent biological replicates are shown. (B) Immunocytochemistry of the SMC proteins CNN1, ACTA2, and SM22A following HESC-derived NC differentiation to SMC with PDGF-BB ( $10 \mathrm{ng} / \mathrm{mL})$ and TGF- $\beta 1$ ( $2 \mathrm{ng} / \mathrm{mL})$ for $12 \mathrm{days}$ (12D PT). White scale bar: $100 \mu \mathrm{m}$, red scale bar: $20 \mu \mathrm{m}$. Representative images of three independent biological replicates. (C) qRT-PCR showing the upregulation of the specific melanocyte genes MITF and KIT and the downregulation of specific NC genes FOXD3 and P75 in NC P7 derived melanocytes (NC-MEL). The relative mRNA level was normalized to the housekeeping gene $P B G D$. Results are presented as mean $\pm \mathrm{SD}$ of three independent experiments. $* P<0.05$; $* * P<0.01$; $* * * P<0.001$. (D) Flow cytometry analysis of CD44 and P75 following differentiation of HESC-derived NC to MSC. Green contour plots represent CD44+P75 double staining in HESC-derived NCs, whereas red contour plots represent the double staining in MSC derived from this population. IgG isotype control staining for P75 and CD44 in both HESC-derived NC and MSC populations are shown as blue and orange contour plots, respectively. MSC derived from NC showed robust expression of CD44 and the absence of P75 expression. Representative results of three independent biological replicates are shown. (E) Alcian blue staining, (F) Oil Red O staining, and (G) Alizarin Red staining, demonstrating chondrocyte, adipocyte, and osteocyte differentiation from HESC-derived NC, respectively. Representative images of three independent biological replicates are shown. White scale bar: $100 \mu \mathrm{m}$, red scale bar: $20 \mu \mathrm{m}$. MSC, mesenchymal stem cells. Color images available online at www.liebertpub.com/scd 

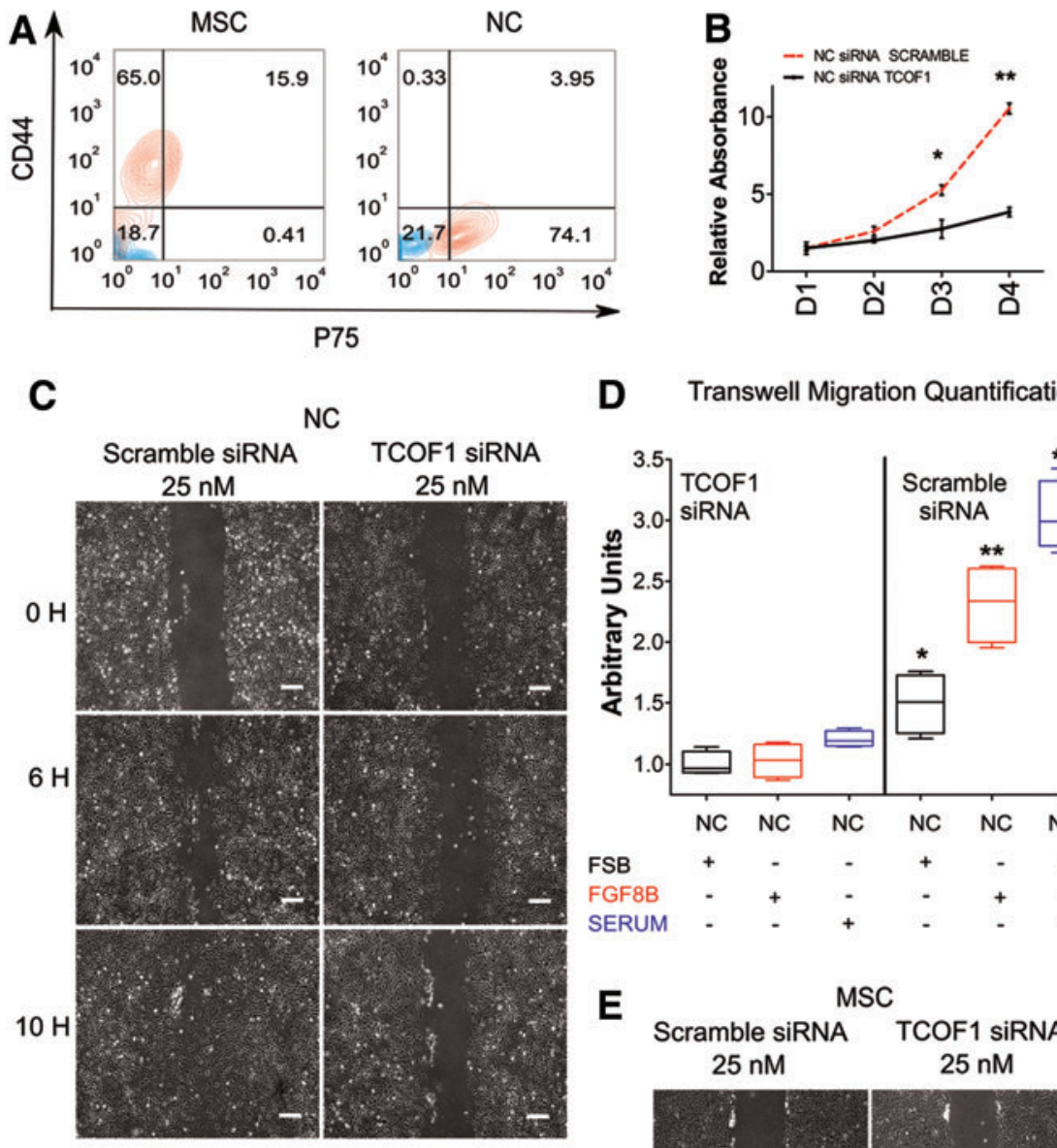

D Transwell Migration Quantification
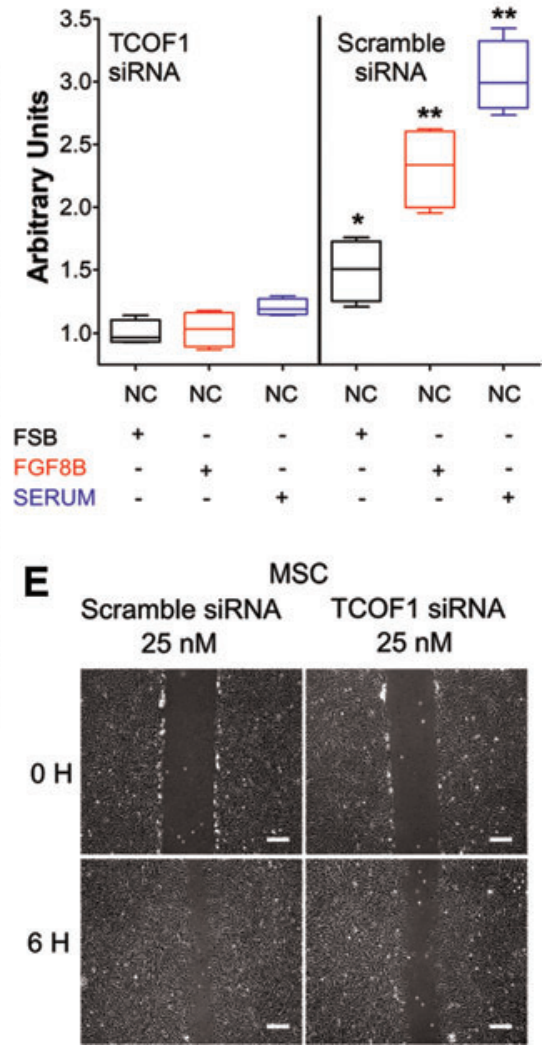

FIG. 6. NC cells transfected with TCOF1 siRNA impair regular migration of NC and MSC. (A) H9s-derived NC cells were transiently transfected with a siRNA to TCOF1. Left panel: Flow cytometry was performed for CD44 and P75 following 5 days of differentiation from NC to MSC, demonstrating that TCOF1 KD does not impair MSC differentiation. Right panel: Flow cytometric analysis of CD44 and P75 in NC transiently transfected with a siRNA to TCOF1. Red contour plots represent CD44+P75 double stained populations, and blue contour plots represent isotype control staining. (B) MTT cell proliferation assay was performed using TCOF1 KD NC cells (NC siRNA TCOF1) and siRNA scramble NC cells (NC siRNA SCRAMBLE) during 4 days. Results are presented as mean $\pm \mathrm{SD}$ of three independent experiments. $* P<0.05$; $* * P<0.01$. Two-sided Student's $t$-test. (C) Representative images of scratch wound assays of HESC-derived NC cells transiently transfected with Scramble siRNA or TCOF1 siRNA. Images were collected 4 days following transfection, at the indicated time points. Scale bar: $100 \mu \mathrm{m}$. (D) Box plot depicting the quantification of chemotaxis potential and migration of NC cells transfected with TCOF1 siRNA or Scramble siRNA during a $6 \mathrm{~h}$ CytoSelect Cell Migration Assay. FGF8B was used as a NC chemoattractant. DMEM/F12+10\% fetal bovine serum (SERUM) was used as a positive control for cell migration. Data are expressed relative to the NC cells transfected with TCOF1 siRNA, maintained in FSB medium. $* P<0.05 ; * * P<0.01$. Two-sided Student's $t$-test. (E) Representative images of scratch wound assays of NC-derived MSC transiently transfected with Scramble siRNA or TCOF1 siRNA. Images were collected after 5 days of differentiation, at the indicated time points. Scale bar: $100 \mu \mathrm{m}$. KD, knockdown; MTT, 3-(4, 5-dimethylthiazolyl-2)-2, 5-diphenyltetrazolium bromide; siRNA, small interfering RNA. Color images available online at www.liebertpub.com/scd

(Fig. 6C). Furthermore, while the migratory defect in this assay could be attributed to the documented impaired proliferation (Fig. 6B), the migration phenotype was detected as early as $6 \mathrm{~h}$ postscratch, suggesting that the phenotype was indeed due to impaired migration.
We performed further validation of this migratory defect using a CytoSelect transwell cell migration assay (Fig. 6D). siRNA-mediated knockdown of TCOF1 produced a significant reduction in NC migration compared with controls (Fig. 6D). This migration impairment was observed in FSB 
medium alone and observed in response to different $\mathrm{NC}$ chemoattractors such as FGF8B [19] or $10 \%$ fetal bovine serum (Fig. 6D). NC-derived MSC transfected with siRNA against TCOF1 also showed a similar migratory defect in a wound healing scratch assay (Fig. 6E). Taken together, these findings demonstrate that TCOF1 may play a role in both the proliferation and migration of the human $\mathrm{NC}$.

\section{Modeling TCS in vitro with TCOF1 ${ }^{+/}$HIPSC using CRISPR/CAS9 technology}

To further confirm the results observed using TCOF1 siRNA in our NC model and to try to model TCS in vitro, we created heterozygous TCOF $1^{+/-}$KO HIPSC lines using CRISPR/Cas9 technology to accurately target Exon 1 of the TCOF1 gene in wild-type (WT) HIPSC (Fig. 7A). Cas9induced double-strand breaks in TCOF1 genomic DNA were repaired by the nonhomologous end joining pathway leading to insertions or deletions (INDELs) in different HIPSC clones (Fig. 7B).

HIPSC Clones 12 and 24 (C12 and C24) had the same single nucleotide deletion in Exon 1 as observed by Sanger sequencing. This removal created a premature stop codon, resulting in a heterozygous $\mathrm{KO}$ for TCOF1 (Fig. 7B). HIPSC Clone 8 (C8) was also transfected, but no INDELs were detected in the TCOF1 gene (data not shown). We, therefore, used $\mathrm{C} 8$ as an isogenic WT HIPSC line to compare with the $\mathrm{TCOF}^{+/-}$clones $(\mathrm{C} 12$ and $\mathrm{C} 24)$. NC was generated from all three clones, and NC P7 cells were used for subsequent studies. Immunoblotting for Treacle confirmed a significant reduction of protein levels in $\mathrm{NC}$ derived from $\mathrm{TCOF}^{+/-}$HIPSC clones (C12 and C24) compared with isogenic WT clone $8 \mathrm{NC}$ and WT HESC-derived NC (Fig. 7C).

The downstream effect of defective ribosomal biosynthesis in mutant $\mathrm{TCOF}^{+/-}$mice is a lower proliferative index than WT NC cells [32]. $\mathrm{TCOF}^{+/-} \mathrm{NC}$ derived from clones $\mathrm{C} 12$ and $\mathrm{C} 24$ had significantly lower proliferation rate than similar WT NC cells derived from the $\mathrm{C} 8$ line and WT HESC, validating the findings observed in the $\mathrm{TCOF} 1^{+/-}$ mouse (Fig. 7D).

The underlying cause of the reported craniofacial anomalies in the $\mathrm{TCOF}^{+/-}$mice is increased apoptosis in the neuroepithelium [32]. This developmental stage overlaps with the period of NC induction and migration from the neural plate border during embryogenesis [27]. To investigate whether our in vitro TCS model recapitulated this phenotype, we differentiated WT and $\mathrm{TCOF}^{+/-}$HIPSC lines to neuroectoderm [34]. Flow cytometric analyses for Annexin $\mathrm{V}$ confirmed a marked induction of apoptosis in neuroectoderm cells derived from $\mathrm{TCOF}^{+/-}$HIPSC (C12 and C24) compared with neuroectoderm derived from WT HIPSC Clone 8 or parental WT HESC (Fig. 7E).

As we unveiled a previously unreported migratory defect in NC and NC-derived MSC deficient for TCOF1 by siRNA (Fig. 6C, E), we performed the same analysis on the generated $\mathrm{TCOF}^{+/-}$lines. A wound healing scratch assay demonstrated impaired migration in both NC (Supplementary Fig. S6A) and MSC (Supplementary Fig. S6B) derived from $\mathrm{TCOF}^{+/-}$HIPSC C24. To further investigate this migratory defect uncovered by the wound healing scratch assay, we performed single cell analysis of cell migration and direc- tionality of movement using real-time imaging of cells over a 12-h time course.

This analysis demonstrated that while WT HIPSC Clone 8 derived NC cells migrated freely in multiple directions over a 12 -h period, $\mathrm{TCOF}^{+/-}$HIPSC derived NC cell shows considerable impairment of migration at a single cell level (Fig. 7F). These findings further validate the reduced migration phenotype of TCOF1-deficient NC cells in the human context.

\section{Discussion}

This study first describes a simple and efficient method of generating NC cells from HPSC using CDM. Importantly, this in vitro system has great potential for investigating the molecular and cellular defects in neurocristopathies such as TCS. We have established a model of TCS using CRISPR/ Cas9 gene editing and identified novel NC and MSC migration defects in our in vitro model.

\section{Development of a differentiation protocol to generate NC from HPSC using a CDM}

Several methods have been reported to produce NC from HPSC, although further work is required to allow the production of cells in a fully CDM and to reduce the complexity of the differentiation conditions. The limitations of the previously reported protocols include the use of biological substrates such as Geltrex ${ }^{\mathrm{TM}}$ [21] or Matrigel [23] that display original batch to batch variability. Other protocols used media enriched with complex supplements such as $\mathrm{N} 2^{\mathrm{TM}}$ and $\mathrm{B} 27^{\mathrm{TM}}[23,43]$.

We have demonstrated that we can generate NC cells from HPSC using minimal cytokines compared with previous protocols [18-21] or WNT pathway activation [23]. This reduction of cytokines makes our protocol simpler and more economically viable. Furthermore, we do not need to perform cell sorting to generate our populations, as we have observed that serial passaging effectively purifies the populations generated.

We have also demonstrated that our cells can undergo freeze-thawing with up to $90 \%$ recovery rates and viability, permitting these cells to be used to generate stocks of NC cells at the same passages (Supplementary Fig. S4J). We have used thawed NC cells for multiple differentiations and have not seen any alterations in their maintenance or differentiation capacity following thawing. Therefore, our protocol permits the generation of a high number of $\mathrm{NC}$ cells which can be banked and used at later dates.

The differentiation of NC from HPSC using this approach recapitulates, to some extent, the normal embryonic developmental stages. In our protocol, the passage of neuroepithelial neuroectoderm at low density in FSB appeared to select the NC population in vitro. The genome-wide mRNA expression studies suggested that earlier passages of $\mathrm{NC}$ populations were a mixed population of neural progenitor cells and premigratory NC cells. Premigratory NC genes such as $\mathrm{RHOB}, \mathrm{CDH}$, and $F O X D 3$ and neural progenitor genes such as AXIN2 [55], LHX2 [91], DVL2 [92], and OLIG2 [93] were upregulated in these populations (Fig. 3B).

It has been previously reported that the patterning of neural versus NC lineages is based on the plating density of 


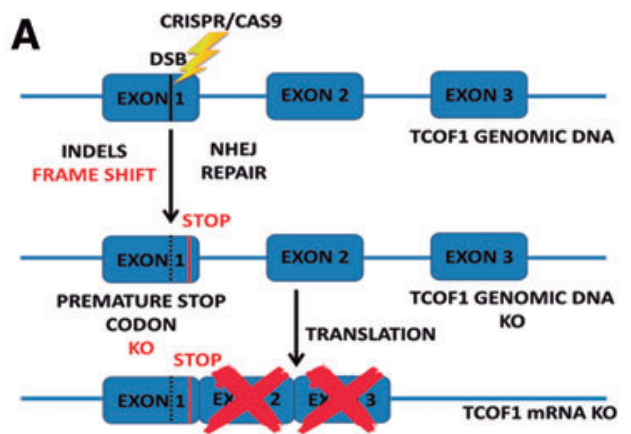

B

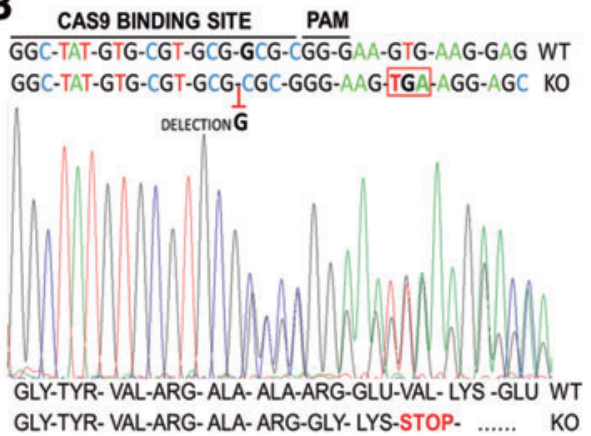

C
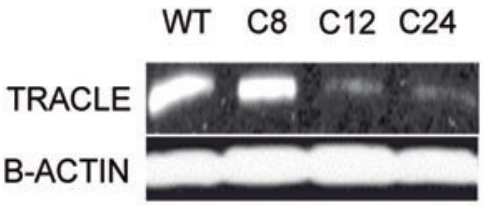

D

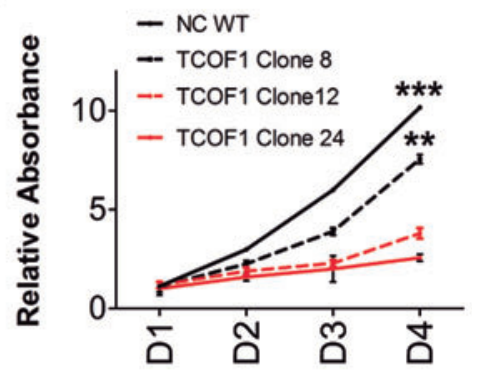

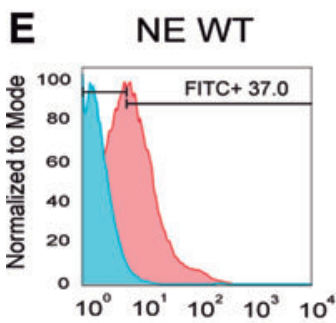
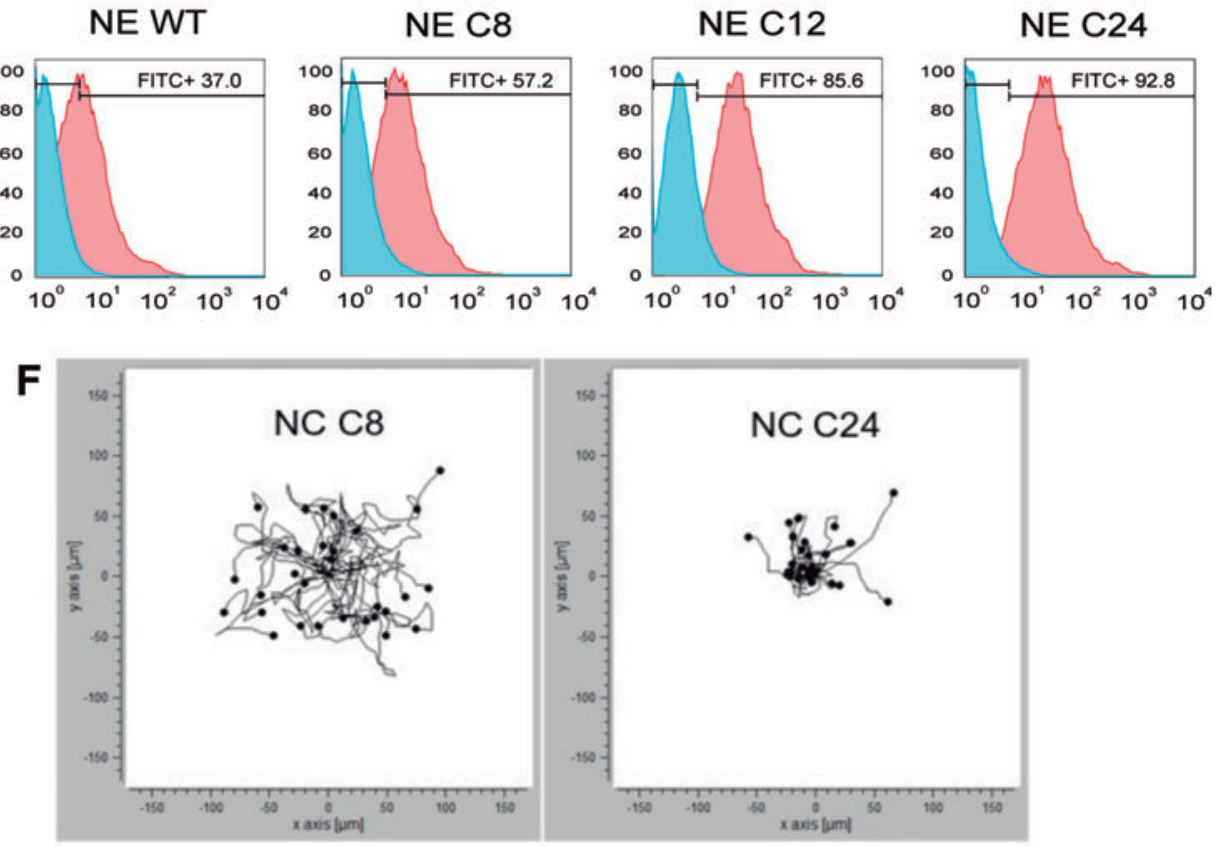

FIG. 7. Generation of TCOF1 heterozygous knockout HIPSC using CRISPR/Cas9. (A) Schematic of CRISPR/Cas9mediated nonhomologous end joining strategy to generate INDELs leading to HIPSC TCOF1 knockout clones. (B) Representative genomic sequencing of HIPSC transfected with specific CRISPR TCOF1 gRNA. Heterozygous knockout clones showed the same deletion leading to a change in codon reading frame and the introduction of a premature STOP codon in TCOF1 Exon 1. (C) Immunoblot for Treacle protein demonstrating that NC derived from HIPSC TCOF ${ }^{+/-}$clones $^{-12}$ (C12 and C24) showed a reduction in Treacle compared with NC derived from HIPSC TCOF1 ${ }^{+/+}$(C8) and NC derived from H9s (WT). (D) Time course MTT proliferation assay of TCOF1 $1^{+/+} \mathrm{NC}$ derived from H9s (WT), HIPSC (C8), and NC derived from TCOF1 $1^{+/}$HIPSC (C12 and C24), over a 4-day period. Proliferation rate decreased significantly in mutated cells compared with WT. Results are presented as mean \pm SD of three independent experiments. $* * P<0.01, * * * P<0.001$, two-sided Student's $t$-test. (E) Flow cytometric analysis of Annexin V staining depicting the apoptotic rate in TCOF1 $1^{+/+}$ NE-derived cells from H9s (WT), HIPSC (C8), and NE derived from TCOF1 ${ }^{\text {t/- }}$ HIPSC (C12 and C24). Red histograms represent Annexin V staining, and blue histograms represent the unstained population. (F) Single cell analysis of cell migration in $\mathrm{NC}$ derived from TCOF $1^{+/+}$HIPSC (C8) and TCOF $1^{+/-}$HIPSC (C24). TCOF $1^{+/-}$NC demonstrates impaired migration and reduced directionality of movement. Each dot and tail represent a single cell analyzed, with 30 cells analyzed from both conditions, over a 12-h period. gRNA, guide RNA; HIPSC, human induced pluripotent stem cell; INDELs, insertions or deletions; WT, wild type. Color images available online at www.liebertpub.com/scd 
early progenitors [18]. Serial passage of our cells at low density selected against the neural population with a significant downregulation of neural genes by later passages (P7). Meanwhile, the P7 cells expressed markers of migratory NC cells, including $S O X 9, S O X 10, P 75$, and TWIST, and downregulated premigratory markers such as WNT1, $P A X 3$, and $R H O B$.

Further validation of in vitro selection upon serial passaging is provided by the pattern of expression of $S O X 10$ and WNT1. In the mouse, Sox10 expression follows Wnt1 expression and marks virtually all NC cells immediately after their delamination from the neural tube [94]. In our system, WNT1 is lowly expressed in mixed NC population, while it increases alongside SOX10 expression in further passages, confirming NC purification in vitro with passaging.

In contrast to other in vitro protocols [18,19,21,43], we did not need to modulate WNT or BMP signaling exogenously. We hypothesized that the endogenous activation of WNT and BMP signaling, as evidenced by our microarray analysis, was sufficient in early passages to induce NC differentiation. This endogenous signaling may reflect the cross-talk and local gradients between a mixed population of the neural plate and nonneural plate ectoderm. Upon further passage of the mixed NC P2 population, we observed downregulation of both BMP and WNT signaling cells in these populations, suggesting that the combination of endogenous BMP and WNT signaling at the early stages of the differentiation protocol was sufficient to specify NC differentiation. The protocol imitates, in part, the steps involved in the development of NC cells in the embryo [9].

The properties of NC cells generated with this protocol were broadly characterized both in vitro and in vivo. We demonstrated the expression of NC markers from passage 7 to beyond. NC cells could proliferate, self-renew, and migrate to appropriate cues such as FGF8B, which has previously been reported [54]. Furthermore, we successfully demonstrated that these NC cells differentiate into their derivative lineages, including MSC, melanocytes, peripheral neurons, SMC, adipocytes, chondrocytes, and osteocytes (Supplementary Fig. S4) [21,23,95].

Importantly, in vivo characterization provides a critical test of a progenitor population's developmental capacity. To determine whether HPSC derived NC cells demonstrated functionality in vivo, we evaluated the integration and migration of these cells in the developing chick embryo. We found the engrafted NC cells localized in the wall of the ascending aorta (Fig. 4), the aortic arch, and the meningeal vessels of the brain (Supplementary Fig. S3).

The absence of NC cells in the epicardium and subepicardial regions of the heart confirmed the specificity of these cells in localizing correctly to $\mathrm{NC}$ in vivo developmental location. There could be several factors that mediate the migration and localization of human NC cells in vivo, including paracrine signals from chicken NC-derived tissues or cell-cell and cell-matrix interactions at particular NCderived locations.

While we have demonstrated that our HIPSC-derived NC can contribute to the aortic SMC population in vivo, we have not explored the broad differentiation of these cells using this approach. The ability to transplant HIPSC-derived cells in vivo provides an opportunity to further our understanding of the developmental potential of human progenitor cells within an in vivo context. Analysis of NC, neuron/ glial, and pericyte marker expression in the transplanted cells may provide additional insight into the differentiation potential of these cells in vivo and their cell fate.

\section{Modeling Treacher Collins syndrome in vitro}

The ability to generate NC from patient-derived HIPSC lines provides a unique tool to study neurocristopathies in the affected cell lineage. A complementary approach to study specific diseases is the de novo creation of a particular mutation in WT lines of HPSC using CRISPR/Cas9 gene editing. This method permits the investigation of multiple disease causing mutations, without the requirement of patient tissue. Moreover, this approach removes the influence played by various genetic backgrounds from different patient samples.

A vast number of NC-related diseases have been reported in the literature, including TCS, although studies using human models are lacking. Treacle protein depletion has been attempted previously using knock-in of a destabilization domaintagging TCOF1 in HEK293 cells, resulting in impaired cell proliferation [87]. Furthermore, mice haploinsufficient for Treacle have been reported and demonstrate robust developmental NC phenotypes [32].

To finally confirm the quality and utility of the HPSCderived NC using this protocol we attempted to model TCS in vitro. We created TCOF $1^{+/-}$HIPSC lines from WT cells using CRISPR/Cas9 technology. The differentiation of $\mathrm{TCOF}^{+/-}$HIPSC clones with our protocol provided the opportunity to study the functionality of the affected $\mathrm{NC}$ cells. TCOF $1^{+/-}$HIPSC derived NC cells displayed significant depletion of Treacle protein, and their phenotype corresponded to the mutant phenotype found in the TCOF $1^{+/-}$ mouse model [32].

Furthermore, neuroectoderm derived from $\mathrm{TCOF}^{+/-}$ HIPSC showed a dramatic increase in apoptosis, replicating the aberrant cell death in the neural plate in $\mathrm{TCOF} 1^{+/-}$mice [32]. The haploinsufficiency of Treacle also leads to reduced proliferation of NC cells in $\mathrm{TCOF}^{+/-}$mice [32]. NC derived from $\mathrm{TCOF}^{+/-}$HIPSC also showed a significant reduction in proliferation compared with WT HIPSC-derived NC cells. Strikingly, we also demonstrated a previously unappreciated impairment in the migration of both $\mathrm{TCOF} 1^{+/-} \mathrm{NC}$ and $\mathrm{NC}$ derived MSC in our in vitro model. Models of TCS have been reported in both the zebrafish and the mouse [32,96]. Both systems demonstrate extensive craniofacial abnormalities, yet the developing NC populations are shown to migrate and populate the maxillary and frontonasal regions normally.

It is of interest to note that while there is no reported difference in the migration behavior of the $\mathrm{NC}$, there is an overall reduction in the number of migrating $\mathrm{NC}$ cells, and this has been demonstrated to be due to a proliferation and cell death defect at the early stages of NC delamination from the neuroepithelium. However, it is possible that the finding of fewer migrating $\mathrm{NC}$ cells in this model may also be due to a proportion of cells which fail to migrate, in addition to the clear cell survival defects reported [32,97]. While we report a migration defect in our TCOF $1^{+/}$HIPSC-based NC system, it is important to acknowledge that this is an in vitro model and so is limited by the many missing developmental cues and triggers which are present in vivo. Therefore, it is possible that the migratory phenotype we report in this study may not be recapitulated during human in vivo development. 
Further engraftment studies using $\mathrm{TCOF}^{+/-}$HIPSCderived NC within the cranial NC may provide novel insights into the mechanisms involved during the migration of the human NC and their contribution to cells of the facial prominences and subsequent craniofacial compartments. Nevertheless, our TCOF $1^{+-}$HIPSC-based NC model now adds to the limited information available regarding human $\mathrm{NC}$ development and its contribution to TCS.

\section{Conclusion}

We believe that this protocol will serve as a resource for researchers seeking to produce $\mathrm{NC}$ and NC-derived tissues and to model neurocristopathies in vitro. Furthermore, we have shown that NC cells derived from HPSC can be differentiated into a wide variety of cell lineages which are of great interest to the field. The ability to study the mechanisms of NC biology and disease should also accelerate the development of innovative therapies to treat, or even prevent, NC disorders such as TCS.

\section{Acknowledgments}

The authors are grateful to Dr. Anna Osnato, Mr. Muhammed Kaiser Abdul Karim, and Dr. Mark Kotter for the provision of cDNA samples and to Dr. Alessandro Bertero for assistance in confocal imaging. The authors are also greatful to Mrs. Nikki Figg and Mr. Alex Petchey for assistance with the sectioning and staining of chicken embryos. This work was supported by the British Heart Foundation (FS/13/29/ 30024, FS/11/77/ 29327, RM/13/3/30159), the British Heart Foundation Cambridge Centre for Research Excellence, and the Stroke Association (TSA2016/02 PP11_Sinha). Core support was provided by the Wellcome-MRC Cambridge Stem Cell Institute (203151/Z/16/Z).

\section{Author Disclosure Statement}

The authors declare no competing financial interests.

\section{References}

1. Le Douarin NM, E Dupin, A Baroffio and C Dulac. (1992). New insights into the development of neural crest derivatives. Int Rev Cytol 138:269-314.

2. Dupin E and L Sommer. (2012). Neural crest progenitors and stem cells: from early development to adulthood. Dev Biol 366:83-95.

3. Morrison SJ, PM White, C Zock and DJ Anderson. (1999). Prospective identification, isolation by flow cytometry, and in vivo self-renewal of multipotent mammalian neural crest stem cells. Cell 96:737-749.

4. Yoshida S, S Shimmura, N Nagoshi, K Fukuda, Y Matsuzaki, H Okano and K Tsubota. (2006). Isolation of multipotent neural crest-derived stem cells from the adult mouse cornea. Stem Cells 24:2714-2722.

5. Zhao C, V Andreeva, Y Gibert, M LaBonty, V Lattanzi, S Prabhudesai, Y Zhou, L Zon, KL McCann, S Baserga and PC Yelick. (2014). Tissue specific roles for the ribosome biogenesis factor Wdr43 in zebrafish development. PLoS Genet 10:e1004074.

6. Southard-Smith EM, L Kos and WJ Pavan. (1998). Sox10 mutation disrupts neural crest development in Dom Hirschsprung mouse model. Nat Genet 18:60-64.
7. Itasaki $\mathrm{N}$ and S Hoppler. (2010). Crosstalk between Wnt and bone morphogenic protein signaling: a turbulent relationship. Dev Dyn 239:16-33.

8. Villanueva S, A Glavic, P Ruiz and R Mayor. (2002). Posteriorization by FGF, Wnt, and retinoic acid is required for neural crest induction. Dev Biol 241:289-301.

9. Liu JP and TM Jessell. (1998). A role for rhoB in the delamination of neural crest cells from the dorsal neural tube. Development 125:5055-5067.

10. Chen ZF and RR Behringer. (1995). twist is required in head mesenchyme for cranial neural tube morphogenesis. Genes Dev 9:686-699.

11. Britsch S, DE Goerich, D Riethmacher, RI Peirano, M Rossner, KA Nave, C Birchmeier and M Wegner. (2001). The transcription factor Sox10 is a key regulator of peripheral glial development. Genes Dev 15:66-78.

12. Moscatelli I, E Pierantozzi, A Camaioni, G Siracusa and L Campagnolo. (2009). p75 neurotrophin receptor is involved in proliferation of undifferentiated mouse embryonic stem cells. Exp Cell Res 315:3220-3232.

13. Matthews HK, L Marchant, C Carmona-Fontaine, S Kuriyama, J Larraín, MR Holt, M Parsons and R Mayor. (2008). Directional migration of neural crest cells in vivo is regulated by Syndecan-4/Rac1 and non-canonical Wnt signaling/RhoA. Development 135:1771-1780.

14. Kuriyama S and R Mayor. (2008). Molecular analysis of neural crest migration. Philos Trans R Soc Lond B Biol Sci 363:1349-1362.

15. Nikitina N, T Sauka-Spengler and M Bronner-Fraser. (2009). Chapter 1 . Gene regulatory networks in neural crest development and evolution. Curr Top Dev Biol 86:1-14.

16. Vallier L, D Reynolds and RA Pedersen. (2004). Nodal inhibits differentiation of human embryonic stem cells along the neuroectodermal default pathway. Dev Biol 275:403-421.

17. Smith JR, L Vallier, G Lupo, M Alexander, WA Harris and RA Pedersen. (2008). Inhibition of Activin/Nodal signaling promotes specification of human embryonic stem cells into neuroectoderm. Dev Biol 313:107-117.

18. Chambers SM, CA Fasano, EP Papapetrou, M Tomishima, M Sadelain and L Studer. (2009). Highly efficient neural conversion of human ES and iPS cells by dual inhibition of SMAD signaling. Nat Biotechnol 27:275-280.

19. Kreitzer FR, N Salomonis, A Sheehan, M Huang, JS Park, MJ Spindler, P Lizarraga, WA Weiss, PL So and BR Conklin. (2013). A robust method to derive functional neural crest cells from human pluripotent stem cells. Am J Stem Cells 2:119-131.

20. Lee G, H Kim, Y Elkabetz, GAl Al Shamy, G Panagiotakos, T Barberi, V Tabar and L Studer. (2007). Isolation and directed differentiation of neural crest stem cells derived from human embryonic stem cells. Nat Biotechnol 25: 1468-1475.

21. Menendez L, MJ Kulik, AT Page, SS Park, JD Lauderdale, ML Cunningham and S Dalton. (2013). Directed differentiation of human pluripotent cells to neural crest stem cells. Nat Protoc 8:203-212.

22. Huang M, ML Miller, LK McHenry, T Zheng, Q Zhen, S Ilkhanizadeh, BR Conklin, ME Bronner and WA Weiss. (2016). Generating trunk neural crest from human pluripotent stem cells. Sci Rep 6:19727.

23. Leung AW, B Murdoch, AF Salem, MS Prasad, GA Gomez and MI García-Castro. (2016). WNT/beta-catenin signaling mediates human neural crest induction via a pre-neural border intermediate. Development 143:398-410. 
24. Fattahi F, JA Steinbeck, S Kriks, J Tchieu, B Zimmer, S Kishinevsky, N Zeltner, Y Mica, W El-Nachef, et al. (2016). Deriving human ENS lineages for cell therapy and drug discovery in Hirschsprung disease. Nature 531:105109.

25. Lee G, EP Papapetrou, H Kim, SM Chambers, MJ Tomishima, CA Fasano, YM Ganat, J Menon, F Shimizu, et al. (2009). Modelling pathogenesis and treatment of familial dysautonomia using patient-specific iPSCs. Nature 461:402-406.

26. Cohen MA, KJ Wert, J Goldmann, S Markoulaki, Y Buganim, D Fu and R Jaenisch. (2016). Human neural crest cells contribute to coat pigmentation in interspecies chimeras after in utero injection into mouse embryos. Proc Natl Acad Sci U S A 113:1570-1575.

27. Poswillo D. (1975). The pathogenesis of the Treacher Collins syndrome (mandibulofacial dysostosis). Br J Oral Surg 13:1-26.

28. Benish BM. (1975). Letter: "The neurocristopathies: a unifying concept of disease arising in neural crest development." Hum Pathol 6:128.

29. Conte C, MR D'Apice, F Rinaldi, S Gambardella, F Sangiuolo and G Novelli. (2011). Novel mutations of TCOF1 gene in European patients with Treacher Collins syndrome. BMC Med Genet 12:125.

30. (1996). Positional cloning of a gene involved in the pathogenesis of Treacher Collins syndrome. The Treacher Collins Syndrome Collaborative Group. Nat Genet 12:130 136.

31. Valdez BC, D Henning, RB So, J Dixon and MJ Dixon. (2004). The Treacher Collins syndrome (TCOF1) gene product is involved in ribosomal DNA gene transcription by interacting with upstream binding factor. Proc Natl Acad Sci U S A 101:10709-10714.

32. Dixon J, NC Jones, LL Sandell, SM Jayasinghe, J Crane, JP Rey, MJ Dixon and PA Trainor. (2006). Tcof1/Treacle is required for neural crest cell formation and proliferation deficiencies that cause craniofacial abnormalities. Proc Natl Acad Sci U S A 103:13403-13408.

33. Rashid ST, S Corbineau, N Hannan, SJ Marciniak, E Miranda, G Alexander, I Huang-Doran, J Griffin, L AhrlundRichter, et al. (2010). Modeling inherited metabolic disorders of the liver using human induced pluripotent stem cells. J Clin Invest 120:3127-3136.

34. Cheung C, AS Bernardo, RA Pedersen and S Sinha. (2014). Directed differentiation of embryonic origin-specific vascular smooth muscle subtypes from human pluripotent stem cells. Nat Protoc 9:929-938.

35. Vallier L, M Alexander and R Pedersen. (2007). Conditional gene expression in human embryonic stem cells. Stem Cells 25:1490-1497.

36. Iyer D, L Gambardella, WG Bernard, F Serrano, VL Mascetti, RA Pedersen, S Sinha and A Talasila. (2016). Robust derivation of epicardium and its differentiated smooth muscle cell progeny from human pluripotent stem cells. Development 143:904.

37. Cheung C, AS Bernardo, MW Trotter, RA Pedersen and S Sinha. (2012). Generation of human vascular smooth muscle subtypes provides insight into embryological origin-dependent disease susceptibility. Nat Biotechnol 30: 165-173.

38. Fang D, K Leishear, TK Nguyen, R Finko, K Cai, M Fukunaga, L Li, PA Brafford, AN Kulp, et al. (2006). Defining the conditions for the generation of melanocytes from human embryonic stem cells. Stem Cells 24:16681677.

39. Heng BC, CM Cowan and S Basu. (2009). Comparison of enzymatic and non-enzymatic means of dissociating adherent monolayers of mesenchymal stem cells. Biol Proced Online 11:161-169.

40. Ran FA, PD Hsu, J Wright, V Agarwala, DA Scott and F Zhang. (2013). Genome engineering using the CRISPRCas9 system. Nat Protoc 8:2281-2308.

41. Choi SY, HC Shin, SY Kim and YW Park. (2008). Role of TMPRSS4 during cancer progression. Drug News Perspect 21:417-423.

42. Szabo R and TH Bugge. (2008). Type II transmembrane serine proteases in development and disease. Int $\mathrm{J}$ Biochem Cell Biol 40:1297-1316.

43. Jung H, KP Lee, SJ Park, JH Park, YS Jang, SY Choi, JG Jung, K Jo, DY Park, et al. (2008). TMPRSS4 promotes invasion, migration and metastasis of human tumor cells by facilitating an epithelial-mesenchymal transition. Oncogene 27:2635-2647.

44. Carney TJ, S von der Hardt, C Sonntag, A Amsterdam, J Topczewski, N Hopkins and M Hammerschmidt. (2007). Inactivation of serine protease Matriptase la by its inhibitor Hail is required for epithelial integrity of the zebrafish epidermis. Development 134:3461-3471.

45. Acloque H, MS Adams, K Fishwick, M Bronner-Fraser and MA Nieto. (2009). Epithelial-mesenchymal transitions: the importance of changing cell state in development and disease. J Clin Invest 119:1438-1449.

46. Suter DM, D Tirefort, S Julien and KH Krause. (2009). A Sox1 to Pax6 switch drives neuroectoderm to radial glia progression during differentiation of mouse embryonic stem cells. Stem Cells 27:49-58.

47. Schorle H, P Meier, M Buchert, R Jaenisch and PJ Mitchell. (1996). Transcription factor AP-2 essential for cranial closure and craniofacial development. Nature 381:235-238.

48. Zhang J, S Hagopian-Donaldson, G Serbedzija, J Elsemore, D Plehn-Dujowich, AP McMahon, RA Flavell and T Williams. (1996). Neural tube, skeletal and body wall defects in mice lacking transcription factor AP-2. Nature 381:238-241.

49. Martinez-Barbera JP, M Signore, PP Boyl, E Puelles, D Acampora, R Gogoi, F Schubert, A Lumsden and A Simeone. (2001). Regionalisation of anterior neuroectoderm and its competence in responding to forebrain and midbrain inducing activities depend on mutual antagonism between OTX2 and GBX2. Development 128:4789-4800.

50. Plouhinec JL, DD Roche, C Pegoraro, AL Figueiredo, F Maczkowiak, LJ Brunet, C Milet, JP Vert, N Pollet, RM Harland and AH Monsoro-Burq. (2014). Pax3 and Zic1 trigger the early neural crest gene regulatory network by the direct activation of multiple key neural crest specifiers. Dev Biol 386:461-472.

51. Monsoro-Burq AH, E Wang and R Harland. (2005). Msxl and Pax3 cooperate to mediate FGF8 and WNT signals during Xenopus neural crest induction. Dev Cell 8:167-178.

52. Cheung M and J Briscoe. (2003). Neural crest development is regulated by the transcription factor Sox9. Development 130:5681-5693.

53. Granata A, F Serrano, WG Bernard, M McNamara, L Low, P Sastry and S Sinha. (2017). An iPSC-derived vascular model of Marfan syndrome identifies key mediators of smooth muscle cell death. Nat Genet 49:97-109.

54. Sato A, AM Scholl, EN Kuhn, HA Stadt, JR Decker, K Pegram, MR Hutson and ML Kirby. (2011). FGF8 signaling 
is chemotactic for cardiac neural crest cells. Dev Biol 354: $18-30$.

55. Kalani MY, SH Cheshier, BJ Cord, SR Bababeygy, H Vogel, IL Weissman, TD Palmer and R Nusse. (2008). Wnt-mediated self-renewal of neural stem/progenitor cells. Proc Natl Acad Sci U S A 105:16970-16975.

56. Pringle NP, WP Yu, M Howell, JS Colvin, DM Ornitz and WD Richardson. (2003). Fgfr3 expression by astrocytes and their precursors: evidence that astrocytes and oligodendrocytes originate in distinct neuroepithelial domains. Development 130:93-102.

57. Tanigaki K, F Nogaki, J Takahashi, K Tashiro, H Kurooka and T Honjo. (2001). Notch1 and Notch3 instructively restrict bFGF-responsive multipotent neural progenitor cells to an astroglial fate. Neuron 29:45-55.

58. Hatou S, S Yoshida, K Higa, H Miyashita, E Inagaki, H Okano, K Tsubota and S Shimmura. (2013). Functional corneal endothelium derived from corneal stroma stem cells of neural crest origin by retinoic acid and Wnt/betacatenin signaling. Stem Cells Dev 22:828-839.

59. Clay MR and MC Halloran. (2014). Cadherin 6 promotes neural crest cell detachment via F-actin regulation and influences active Rho distribution during epithelial-tomesenchymal transition. Development 141:2506-2515.

60. Sela-Donenfeld D and C Kalcheim. (1999). Regulation of the onset of neural crest migration by coordinated activity of BMP4 and Noggin in the dorsal neural tube. Development 126:4749-4762.

61. Dottori M, MK Gross, P Labosky and M Goulding. (2001). The winged-helix transcription factor Foxd3 suppresses interneuron differentiation and promotes neural crest cell fate. Development 128:4127-4138.

62. Mancilla A and R Mayor. (1996). Neural crest formation in Xenopus laevis: mechanisms of Xslug induction. Dev Biol 177:580-589.

63. Le Douarin NM and E Dupin. (1993). Cell lineage analysis in neural crest ontogeny. J Neurobiol 24:146-161.

64. Giovannone D, B Ortega, M Reyes, N El-Ghali, M Rabadi, S Sao and ME de Bellard. (2015). Chicken trunk neural crest migration visualized with HNK1. Acta Histochem 117:255-266.

65. Gajavelli S, PM Wood, D Pennica, SR Whittemore and P Tsoulfas. (2004). BMP signaling initiates a neural crest differentiation program in embryonic rat CNS stem cells. Exp Neurol 188:205-223.

66. Raible DW and JW Ragland. (2005). Reiterated Wnt and BMP signals in neural crest development. Semin Cell Dev Biol 16:673-682.

67. Steventon B, C Araya, C Linker, S Kuriyama and R Mayor. (2009). Differential requirements of BMP and Wnt signalling during gastrulation and neurulation define two steps in neural crest induction. Development 136:771-779.

68. Moon RT, AD Kohn, GV De Ferrari and A Kaykas. (2004). WNT and beta-catenin signalling: diseases and therapies. Nat Rev Genet 5:691-701.

69. Milet C and AH Monsoro-Burq. (2012). Neural crest induction at the neural plate border in vertebrates. Dev Biol 366:22-33.

70. Marchant L, C Linker, P Ruiz, N Guerrero and R Mayor. (1998). The inductive properties of mesoderm suggest that the neural crest cells are specified by a BMP gradient. Dev Biol 198:319-329.

71. Liem KF, Jr., G Tremml, H Roelink and TM Jessell. (1995). Dorsal differentiation of neural plate cells induced by BMP-mediated signals from epidermal ectoderm. Cell 82:969-979.

72. Santana MM, KF Chung, V Vukicevic, J RosmaninhoSalgado, W Kanczkowski, V Cortez, K Hackmann, CA Bastos, A Mota, et al. (2012). Isolation, characterization, and differentiation of progenitor cells from human adult adrenal medulla. Stem Cells Transl Med 1:783-791.

73. Yardley N and MI García-Castro. (2012). FGF signaling transforms non-neural ectoderm into neural crest. Dev Biol 372:166-177.

74. Kirby ML, TF Gale and DE Stewart. (1983). Neural crest cells contribute to normal aorticopulmonary septation. Science 220:1059-1061.

75. Goldstein RS, M Drukker, BE Reubinoff and N Benvenisty. (2002). Integration and differentiation of human embryonic stem cells transplanted to the chick embryo. Dev Dyn 225: 80-86.

76. Boulland JL, G Halasi, N Kasumacic and JC Glover. (2010). Xenotransplantation of human stem cells into the chicken embryo. J Vis Exp 41:2071.

77. Sieber-Blum M and SR Patel. (1986). In vitro differentiation of quail neural crest cells into sensory neurons. Prog Clin Biol Res 217B:243-248.

78. Nakajima T, M Ota and K Ito. (2008). Differentiation of autonomic neurons by BMP-independent mechanisms. Cell Tissue Res 332:25-35.

79. Garcez RC, BL Teixeira, Sdos S Schmitt, M Alvarez-Silva and AG Trentin. (2009). Epidermal growth factor (EGF) promotes the in vitro differentiation of neural crest cells to neurons and melanocytes. Cell Mol Neurobiol 29:10871091.

80. Chan AA, AJ Hertsenberg, ML Funderburgh, MM Mann, Y Du, KA Davoli, JD Mich-Basso, L Yang and JL Funderburgh. (2013). Differentiation of human embryonic stem cells into cells with corneal keratocyte phenotype. PLoS One 8:e56831.

81. Billon N, P Iannarelli, MC Monteiro, C GlavieuxPardanaud, WD Richardson, N Kessaris, C Dani and E Dupin. (2007). The generation of adipocytes by the neural crest. Development 134:2283-2292.

82. Akiyama H, JE Kim, K Nakashima, G Balmes, N Iwai, JM Deng, Z Zhang, JF Martin, RR Behringer, T Nakamura and B de Crombrugghe. (2005). Osteo-chondroprogenitor cells are derived from Sox 9 expressing precursors. Proc Natl Acad Sci U S A 102:14665-14670.

83. Kawaguchi J, PJ Mee and AG Smith. (2005). Osteogenic and chondrogenic differentiation of embryonic stem cells in response to specific growth factors. Bone 36:758-769.

84. Jiang X, DH Rowitch, P Soriano, AP McMahon and HM Sucov. (2000). Fate of the mammalian cardiac neural crest. Development 127:1607-1616.

85. Kestendjieva S, D Kyurkchiev, G Tsvetkova, T Mehandjiev, A Dimitrov, A Nikolov and S Kyurkchiev. (2008). Characterization of mesenchymal stem cells isolated from the human umbilical cord. Cell Biol Int 32:724-732.

86. Magdalou J, P Netter, S Fournel-Gigleux and M Ouzzine. (2008). Agrecan and articular cartilage: assessment of glycosyltransferases for the restoration of cartilage matrix in osteoarthritis [in French]. J Soc Biol 202:281-288.

87. Park A, ST Won, M Pentecost, W Bartkowski and B Lee. (2014). CRISPR/Cas9 allows efficient and complete knockin of a destabilization domain-tagged essential protein in a human cell line, allowing rapid knockdown of protein function. PLoS One 9:e95101. 
88. Dacic S, I Kalajzic, D Visnjic, AC Lichtler and DW Rowe. (2001). Colla1-driven transgenic markers of osteoblast lineage progression. J Bone Miner Res 16:1228-1236.

89. Schürmann M, A Wolff, D Widera, S Hauser, P Heimann, A Hütten, C Kaltschmidt and B Kaltschmidt. (2014). Interaction of adult human neural crest-derived stem cells with a nanoporous titanium surface is sufficient to induce their osteogenic differentiation. Stem Cell Res 13:98-110.

90. Katsanis SH and EW Jabs. (1993). Treacher Collins syndrome. In: GeneReviews ${ }^{\circledR}$ [Internet]. Seattle (WA): University of Washington, Seattle; 1993-2018. Available from: https://www.ncbi.nlm.nih.gov/books/NBK1116/

91. Molyneaux BJ, P Arlotta, JR Menezes and JD Macklis. (2007). Neuronal subtype specification in the cerebral cortex. Nat Rev Neurosci 8:427-437.

92. Andersson T, E Södersten, JK Duckworth, A Cascante, N Fritz, P Sacchetti, I Cervenka, V Bryja and O Hermanson. (2009). CXXC5 is a novel BMP4-regulated modulator of Wnt signaling in neural stem cells. J Biol Chem 284:36723681.

93. Ligon KL, E Huillard, S Mehta, S Kesari, H Liu, JA Alberta, RM Bachoo, M Kane, DN Louis, et al. (2007). Olig2regulated lineage-restricted pathway controls replication competence in neural stem cells and malignant glioma. Neuron 53:503-517.

94. Hari L, I Miescher, O Shakhova, U Suter, L Chin, M Taketo, WD Richardson, N Kessaris and L Sommer. (2012). Temporal control of neural crest lineage generation by Wnt/beta-catenin signaling. Development 139:2107-2117.
95. Menendez L, TA Yatskievych, PB Antin and S Dalton. (2011). Wnt signaling and a Smad pathway blockade direct the differentiation of human pluripotent stem cells to multipotent neural crest cells. Proc Natl Acad Sci U S A 108:19240-19245.

96. Weiner AM, NL Scampoli and NB Calcaterra. (2012). Fishing the molecular bases of Treacher Collins syndrome. PLoS One 7:e29574.

97. Sakai D, J Dixon, A Achilleos, M Dixon and PA Trainor. (2016). Prevention of Treacher Collins syndrome craniofacial anomalies in mouse models via maternal antioxidant supplementation. Nat Commun 7:10328.

Address correspondence to:

Dr. Sanjay Sinha

Anne McLaren Laboratory

Wellcome Trust-Medical Research Council Cambridge

Stem Cell Institute

University of Cambridge

Forvie Site, Robinson Way Cambridge CB2 OSZ United Kingdom

E-mail: ss661@cam.ac.uk

Received for publication November 8, 2017

Accepted after revision October 29, 2018

Prepublished on Liebert Instant Online October 30, 2018 